\title{
Use of natural ingredients in Japanese quail diet and their effect on carcass and meat quality - A review
}

\author{
Rey David Vargas-Sánchez ${ }^{1}$, Félix Joel Ibarra-Arias², Brisa del Mar Torres-Martínez ${ }^{1}$, \\ Armida Sánchez-Escalante ${ }^{1}$, and Gastón Ramón Torrescano-Urrutia ${ }^{1, *}$
}

\author{
* Corresponding Author: \\ Gastón Ramón Torrescano-Urrutia \\ Tel: +52-662-2892400 (626) \\ Fax: $+52-662-2800421$, \\ E-mail: gtorrescano@ciad.mx
}

${ }^{1}$ Meat and Meat Products Research Laboratory, Department of Technology of Animal Origin Food, Research Center for Food and Development, A.C. Hermosillo Sonora, ZIP 83304, Mexico

${ }^{2}$ Alta Tecnología Industrial para la Salud Animal, S.A. de C.V. Guadalajara Jalisco, ZIP 44430, Mexico

ORCID

Rey David Vargas-Sánchez

https://orcid.org/0000-0002-8537-1155

Félix Joel Ibarra-Arias

https://orcid.org/0000-0002-0722-2883

Brisa del Mar Torres-Martínez

https://orcid.org/0000-0003-0354-9982

Armida Sánchez-Escalante

https://orcid.org/0000-0002-5966-5512

Gastón Ramón Torrescano-Urrutia

https://orcid.org/0000-0003-1117-501X

Submitted Oct 25, 2018; Revised Nov 21, 2018; Accepted Jan 15, 2019

\begin{abstract}
The present paper reviews the findings of different research studies on the effect of natural ingredients in the Japanese quail (Coturnix coturnix japonica) diet on carcass characteristics and meat quality. The results show a relationship between the type and concentration of ingredients used in diets and carcass characteristics and meat quality. The inclusion of medicinal herbs (thyme, black seed, and mint), plants (canola), seeds (chickpea), spices (cinnamon and coriander), worms (earthworms), bee products (propolis), phytochemicals (lycopene), and edible fungi (common mushrooms) in the diet improved carcass quality characteristics compared to the control diets (basal diets). The inclusion of medicinal herbs (spearmint and green tea), spices (cinnamon), vegetables (tomato), plants (verbena and canola), seeds (marijuana), and edible fungi (oyster mushrooms) improved meat quality. In conclusion, the use of ingredients of natural origin in the diet of Japanese quail improves carcass quality characteristics and meat quality.
\end{abstract}

Keywords: Japanese Quail; Animal Diet; Natural Ingredients; Carcass Quality; Meat Quality

\section{INTRODUCTION}

The production and consumption of poultry meat in Mexico grew at an average annual rate of $3.2 \%$ and $2.7 \%$, respectively, from 2006 to 2015 . Poultry consumption amounted to $32.0 \mathrm{~kg}$ per capita in 2016 [1]. Domestically, 4,061 M metric tons of poultry were consumed in 2016, and 3,275 M metric tons were produced [2]. The increase in both the production and consumption of poultry can be associated with the greater availability of feed grains, low feed costs, and the accessible price of poultry products as well as the increase in the price of other meats such as pork and beef [1]. To meet domestic demand, in 2016, the import of chicken meat increased to $791 \mathrm{M}$ metric tons [2]. These figures reflect an opportunity to produce more chicken meat locally and nationally, including alternative meats from other species (turkey, duck, guinea fowl, goose, ostrich, pheasant, and quail), without disregarding the criteria of accessibility, price, and quality [3].

The Japanese quail (Coturnix coturnix japonica) is native to Europe, northern Africa, and Asia. The raising of Japanese quail has been characterized as economically sustainable and highly productive $[4,5]$, as this quail has a rapid growth cycle (3 to 4 generations per year) and is resistant to diseases [5,6]. With respect to meat production, this bird is considered the smallest of the poultry varieties, making it is easy to manage and to accommodate a large number of birds in a small space. For these reasons, the Japanese quail has also gained importance worldwide for its use in experimental animal models in biological and genetic studies [5]. Therefore, coturniculture, or the raising of quail, is expanding, including the 
commercialization of different quail products such as fresh or pickled eggs and fresh or frozen carcasses $[4,7]$. The carcass and meat of Japanese quail are obtained after the quail reaches 35 to $42 \mathrm{~d}$ of age and a body weight of 165 to $300 \mathrm{~g}$ $[5,8]$. However, data on quail production are limited regarding the quality of carcass meat destined for processing (31 and $138 \mathrm{M}$ carcasses, for America and Europe, respectively), and the available data are lacking in precision [9].

As is the case for other birds, the integration and management of quail production (breeding, slaughter, processing, and marketing) is essential for maintaining carcass and meat quality $[4,5,10]$. During the first stage of production, an initiation and growth diet containing a balanced set of nutrients such as carbohydrates, amino acids, essential fatty acids, minerals, vitamins, and water is fed to animals. The quality of quail carcasses is dependent on an adequate and constant supply of energy. The production of birds in temperate climates requires a total of 2,600 to $3,000 \mathrm{kcal} / \mathrm{kg}$ of metabolizable energy and, in tropical regions, requires $2,800 \mathrm{kcal} / \mathrm{kg}[11,12]$. Dietary sources commonly used to meet the carbohydrate requirement include grains, such as corn, whereas earthworms, fish, and soybean meal are often used as a protein source $[4,8,13$, 14]. In addition, soybean and safflower oils can be used as sources of fatty acids $[8,14]$. To increase the quality of poultry meat, which is mainly affected by oxidative stress, natural ingredients, such as organic compounds (tocopherols), rosemary, green tea, tomato, and honey extracts, have been used in the poultry diet because they contain beneficial compounds with antioxidant activity [15].

Based on the above, the present review summarizes existing research on the inclusion of natural ingredients in the Japanese quail diet to highlight their effect on carcass and meat quality.

\section{CARCASS AND MEAT COMPOSITION}

In relation to chemical composition, the Japanese quail carcass (at 35 to 42 days of age) is composed of $68 \%$ water, $19 \%$ protein, $10 \%$ fat, and $3 \%$ minerals [16]. The meat (breasts and legs) is composed of $71 \%$ to $74 \%$ water, $17 \%$ to $23 \%$ protein, $2 \%$ to $8 \%$ fat, and $1.5 \%$ to $1.8 \%$ minerals $[13,16,17]$. According to this chemical composition, the protein content of both the carcass and meat is like that found in the literature for the carcass $(19.0 \%)$ and the legs and breasts $(20 \%$ and $23 \%$, respectively) of broiler chickens $[18,19]$ but lower than that of red meat (20\% to $25 \%$ ) [20]. In addition, quail meat is considered a valuable source of protein because of its good amino acid profile. Breast and leg meat contain essential amino acids such as cysteine, phenylalanine, isoleucine, leucine, lysine, methionine, tyrosine, threonine, and valine as well as nonessential amino acids such as alanine, arginine, asparagine, glycine, glutamine, histidine, proline, and serine [16]. Differ- ent minerals have also been identified in quail meat, including calcium, phosphorus, sodium, potassium, magnesium, iron, copper, and zinc $[16,21]$. In adequate quantities, these minerals contribute to the formation of the skeletal system and to the health of animals considering that different minerals are involved in metabolic activity and in maintaining the acid-base balance of the body [22].

The fat content of the leg and breast meat of Japanese quails is $3.3 \%$ and $2.5 \%$ respectively [16]. These values are within the range reported for beef (Longissimus dorsi) with light marbling (2.1\% to 3.7\%) but lower than those reported for lamb meat (Longissimus dorsi; $8 \%$ ) $[23,24]$. In quail meat, the lipid profile mainly consists of four fatty acids: oleic (C18:1), palmitic (C16:0), linoleic (C18:2), and stearic (C18:0). These four fatty acids represent around $80 \%$ of the total fatty acid content in the breast and leg meat of Japanese quail, with C18:1 being present in the highest proportion $[16,17,21,25]$. With respect to saturated fatty acids (SFAs), C14:0 presents a higher concentration (18.8\%) in the leg than in the breast [21]. Meanwhile, with respect to polyunsaturated fatty acids (PUFAs), C18:2 is present the highest concentration, representing 20\% of total lipids [16]. On the other hand, the sum of unsaturated fatty acids (UFAs) amounts to $60 \%$ in quail meat $[16,25]$. Additionally, it was reported that birds are able to deposit high amounts of $\alpha$-linolenic acid in meat when their rations are rich in this fatty acid [16] and when the proportions of UFA:SFA, PUFA:SFA, and PUFAn-6:n-3 in breast meat range from 0.39 to $1.92,0.20$ to 0.73 , and 9.31 to 15.3 , respectively $[16,21,25]$. The PUFAn-6:n-3 ratio in meat is considered one of the main evaluation criteria of dietary properties; for broiler meat, a proportion of 16.02 has been reported [26]. The aforementioned ratios of nutritive components are important for maintaining the optimal growth of Japanese quail and for protecting the carcass and meat from oxidative stress $[11,25,27]$. The Table 1 summarized the chemical composition of quail breast meat in comparison with broiler breast meat.

\section{EFFECT OF OXIDATIVE STRESS ON CARCASS AND MEAT QUALITY}

Oxidative stress occurs as a result of the formation of oxidizing agents, the normal products of aerobic metabolism, or as a result of the intake of oxidizing agents through the diet at a rate that exceeds the capacity of the antioxidant system to eliminate reactive oxygen species, causing significant biological damage in animals and, in particular, affecting the growth of birds [30]. The formation of products from oxidative metabolism can be elevated under stressful conditions and during slaughter, affecting animal welfare and the quality characteristics of carcasses and meat, although suitable breeding practices as well as antemortem conditions can improve the physiological or biochemical state of an animal $[30,31]$. 
Table 1. Chemical composition of quail and chicken breast meat (\%)

\begin{tabular}{|c|c|c|c|c|c|c|}
\hline \multirow[b]{2}{*}{ Items } & \multicolumn{4}{|c|}{ Quail meat } & \multicolumn{2}{|c|}{ Chicken meat } \\
\hline & $\begin{array}{c}\text { Genchev et al } \\
{[16]}\end{array}$ & $\begin{array}{c}\text { Hamm and Ang } \\
{[17]}\end{array}$ & $\begin{array}{c}\text { Cullere et al } \\
{[21]}\end{array}$ & $\begin{array}{c}\text { Botsoglou et al } \\
{[25]}\end{array}$ & $\begin{array}{c}\text { Wattanachant et al } \\
{[28]}\end{array}$ & $\begin{array}{c}\text { Santoso et al } \\
{[29]}\end{array}$ \\
\hline \multicolumn{7}{|l|}{ Proximate composition } \\
\hline Dry matter & 26.7 & 25.4 & 24.7 & & 25.1 & 30.1 \\
\hline Protein & 21.8 & 18.9 & 18.4 & & 20.6 & 16.3 \\
\hline Fat & 2.9 & 7.9 & 4.6 & & 0.68 & 7.7 \\
\hline Ash & 1.6 & 0.9 & 1.7 & & 1.1 & \\
\hline \multicolumn{7}{|l|}{ Essential amino acids } \\
\hline Cysteine & 2.16 & & & & 0.31 & \\
\hline Phenylalanine & 0.97 & & 0.53 & & 3.01 & 0.6 \\
\hline Isoleucine & 1.17 & & 1.09 & & 2.41 & 0.9 \\
\hline Leucine & 2.00 & & 1.77 & & 4.29 & 1.4 \\
\hline Lysine & 2.16 & & 0.46 & & 3.41 & 1.3 \\
\hline Methionine & 0.54 & & 0.49 & & 1.88 & 0.4 \\
\hline Tyrosine & 0.58 & & 1.85 & & 3.03 & 0.5 \\
\hline Threonine & 0.72 & & 1.12 & & 3.02 & 0.6 \\
\hline Valine & 1.22 & & 1.34 & & 2.16 & 1.0 \\
\hline \multicolumn{7}{|l|}{ Non-essential amino acids } \\
\hline Alanine & 1.32 & & 1.89 & & 2.8 & 1.2 \\
\hline Arginine & 1.36 & & 1.10 & & 4.39 & 1.1 \\
\hline Asparagine & 1.99 & & 2.20 & & 3.64 & 1.7 \\
\hline Glycine & 1.07 & & 1.73 & & 2.70 & 0.5 \\
\hline Glutamine & 3.89 & & 3.54 & & 6.35 & 3.9 \\
\hline Histidine & 0.92 & & 0.03 & & 2.9 & 0.4 \\
\hline Proline & 0.99 & & 0.92 & & 1.93 & \\
\hline Serine & 0.41 & & 1.18 & & 2.38 & 0.4 \\
\hline \multicolumn{7}{|l|}{ Fatty acids } \\
\hline Myristic (C14:0) & 1.04 & 0.8 & 0.25 & 1.44 & 0.87 & 0.4 \\
\hline Palmitic (C16:0) & 24.5 & 20.6 & 17.8 & 22.1 & 31.8 & 19.9 \\
\hline Palmitoleic (C16:1) & 5.69 & 3.1 & 1.25 & 3.32 & 3.33 & 4.6 \\
\hline Stearic (C18:0) & 8.4 & 7.1 & 14.3 & 12.3 & 14.6 & 3.7 \\
\hline Oleic (C18:1) & 35.5 & 44.8 & 10.2 & 24.8 & 37.8 & 37.2 \\
\hline Linoleic (C18:2) & 20 & 22.9 & & 19.52 & 7.63 & 11.7 \\
\hline Linolenic (C18:3) & 1.61 & 0.1 & & 1.21 & 0.16 & 0.12 \\
\hline Arachidonic (C20:4) & 2.32 & & & 3.08 & 0.45 & 0.39 \\
\hline$\Sigma$ Saturated fatty acids & 33.9 & & 34.6 & 41.6 & 48.8 & 24.2 \\
\hline$\Sigma$ Unsaturated fatty acids & 65.9 & & & & & 55.2 \\
\hline$\Sigma$ Monounsaturated fatty acids & 41.1 & & & 28.6 & 41.82 & \\
\hline$\Sigma$ Polyunsaturated fatty acids & 24.7 & & 42.7 & 26.26 & 9.42 & \\
\hline \multicolumn{7}{|l|}{ Minerals } \\
\hline Calcium & 0.02 & 0.02 & 0.05 & & & 0.015 \\
\hline Phosphorus & 0.22 & 0.15 & 0.89 & & & 0.19 \\
\hline Sodium & 0.07 & 0.06 & 0.25 & & & \\
\hline Potassium & 0.40 & 0.19 & 1.5 & & & 0.015 \\
\hline Magnesium & 0.02 & 0.03 & 0.11 & & & \\
\hline Iron & 1.72 & & $<0.001$ & & & 0.016 \\
\hline Copper & 0.37 & & $<0.001$ & & & \\
\hline Zinc & 2.02 & & $<0.001$ & & & \\
\hline
\end{tabular}

Regarding the stress experienced by Japanese quails before and during slaughter, the age, sex, and genetics of animals in addition to environmental conditions [8,32-34], feeding, transportation, withdrawal of food, and slaughter method have been found to affect this factor $[33,34]$. Stress conditions increase the consumption of glycogen and ATP under essentially anaerobic conditions and accelerate the fall of muscle $\mathrm{pH}[35$, 36], resulting in negative changes to carcass characteristics, 
such as weight loss and reduction in the yield of different cuts of the quail carcass $[8,27]$. Additionally, in quail meat, these negative changes manifest in the physical properties of meat, including the color and texture [37]; the chemical content in terms of moisture, protein, lipids, and ash [16]; the technological properties, such as decreased water holding capacity, increased weight loss during cooking, and greater drip loss $[27,32,37]$; and in the biochemical processes with respect to lipid oxidation [27].

To reduce the effects of oxidative stress, antimicrobial compounds (growth-promoter antibiotics, GPAs), including quinoxalines (carbadox and olaquindox), glycopeptides (avoparcin), ionophores (monensin and salomycin), macrolides (tylosin and spiramycin), phosphoglycolipids (flavomycin), streptogramins (virginiamycin), polypeptides (zinc bacitracin), and oligosaccharides (avilamycin), are commonly included in poultry diets for disease control and growth promotion [38]. However, the European Union has banned GPAs, even food-grade antibiotics, because of the possible risk of human pathogenic bacteria developing greater resistance. In other countries in Latin America, efforts are being made to prohibit their use [38-40]. Notably, the growth promotion mechanisms of GPAs are still unknown, yet different hypotheses have been proposed to explain GPAs mechanisms. In particular, GPAs may i) protect nutrients against bacterial destruction, ii) improve nutrient absorption due to the thinning of the small intestine barrier, and iii) reduce the formation of toxins produced by intestinal bacteria $[38,41]$.

In addition, oxidative stress can increase the formation of reactive oxygen species (ROS), including free radicals (hydroxyl, $\mathrm{HO}^{\circ}$; superoxide, $\mathrm{O}_{2}^{\circ}$; nitric oxide, $\mathrm{NO}^{\circ}$, alkoxy, $\mathrm{RO}^{\circ}$; and peroxide, $\left.\mathrm{ROO}^{\circ}\right)$, which can react with the proteins and lipids of meat and cause the deterioration of meat quality during storage [42]. Therefore, to retard the oxidative process in meat, synthetic antioxidants such as butylated hydroxyanisole, butylated hydroxytoluene, tert-butylhydroquinone, and propyl gallate are used $[42,43]$. However, the use of synthetic antioxidants has been associated with potential health risks (e.g., carcinogenesis), which has promoted the establishment of strict regulations in the European Union (Directive of the European Parliament and of the Council No.95/2/EC, dated February 20, 1995) to control their use in foods $[43,44]$.

For this reason, different alternatives are being investigated to improve carcass and meat characteristics, including new nutritional strategies based on the use of natural ingredients (e.g., medicinal herbs, fruits, and plants). Some of the compounds contained in natural ingredients have antimicrobial and antioxidant properties that may exert an effect on animals like those of GPAs and synthetic antioxidants. Their use can possibly reduce stress caused by inadequate management practices during the production and slaughter of birds as well as improve meat quality by increasing oxidative sta- bility through the antioxidant system $[6,15,44]$.

\section{NATURAL ADDITIVES FOR ENHANCING CARCASS AND MEAT QUALITY}

According to the guidelines of the Codex Alimentarius Commission [45], a food additive is "any substance that as such is not normally consumed as a food, nor is it used as a basic ingredient in food, whether or not it has a nutritional value, and whose intentional addition to food for technological purposes (including organoleptic) in its manufacturing, processing, preparation, treatment, packaging, transport, storage or storage phases, is reasonably expected to result (directly or indirectly) by itself or its by-products, in one component of food or an element that affects its characteristics. This definition does not include contaminants or substances added to the food to maintain or improve nutritional qualities."

On the other hand, the FAO [46] establishes that feed (animal feed) is "all simple or compound material, whether processed, semi-processed or unprocessed, used directly in feeding animals intended for human consumption". In addition, it defines feed ingredient as a "part or constituent of any combination or mixture that constitutes a feed, whether or not has a nutritional value in animal feed, including feed additives". Furthermore, ingredients can be "substances of vegetable, animal or aquatic origin, or other organic or inorganic substances". Meanwhile, a feed additive is considered "any deliberately added ingredient that is not normally consumed as feed, whether it has a nutritional value, and that influences the characteristics of the feed or animal products".

According to the National Research Council [11], to cover the basal diet requirements of Japanese quail (metabolizable energy $=2,900 \mathrm{kcal} / \mathrm{kg}$ ), certain nutrients must be included in the diet during the initiation and growth stage, such as i) proteins $(24 \%)$ and amino acids, specifically arginine (1.25\%), glycine+serine (1.25\%), histidine $(0.36 \%)$, isoleucine $(0.98 \%)$, leucine $(1.69 \%)$, lysine $(1.30 \%)$, methionine $(0.50 \%)$, methionine+cysteine $(0.75 \%)$, phenylalanine $(0.96 \%)$, phenylalanine +tyrosine (1.80\%), threonine (1.02\%), tryptophan (0.22\%), and valine $(0.95 \%)$; ii) fat and macro minerals such as linoleic acid $(1.0 \%)$, calcium $(0.8 \%)$, chlorine $(0.14 \%)$, magnesium (300 mg), non-phytate phosphorus $(0.3 \%)$, potassium $(0.4 \%)$, and sodium (0.15\%); iii) trace minerals such as copper (5 mg), iodine $(0.3 \mathrm{mg})$, iron $(120 \mathrm{mg})$, manganese $(60 \mathrm{mg})$, selenium (0.2 $\mathrm{mg})$, and zinc ( $25 \mathrm{mg})$; and iv) fat-soluble vitamins such as vitamin A (1,650 IU), vitamin D3 (750 ICU), vitamin E (12 $\mathrm{IU})$, and vitamin $\mathrm{K}(1 \mathrm{mg})$ and water-soluble vitamins such as vitamin $B_{12}(0.003 \mathrm{mg})$, biotin $(2 \mathrm{mg})$, folic acid (1 mg), niacin (40 $\mathrm{mg})$, pantothenic acid (10 $\mathrm{mg})$, pyridoxine (3 $\mathrm{mg})$, riboflavin (4 mg), and thiamine (2 mg).

Although the abovementioned basic diet is required for the optimal growth of Japanese quail, as confirmed by numerous 
investigations, it may be possible to increase the yield of carcasses, mainly through reducing the infectious agents that affect the growth and quality of carcasses. Table 2 lists the effects of natural additives in the quail diet on carcasses, as

Table 2. Carcass characteristics of Japanese quail supplemented with natural ingredients in their diet

\begin{tabular}{|c|c|c|c|}
\hline Natural ingredients & Bird management & Relevant results & References \\
\hline \multicolumn{4}{|l|}{ Medicinal herbs } \\
\hline $\begin{array}{l}\text { Thyme (Thymus vulgaris L.) } \\
\text { and black seed } \\
\text { (Nigella sativa L.) }\end{array}$ & $\begin{array}{l}\text { Age and weight: } 1 \mathrm{~d} \text {-old/N.A. } \\
\text { Dose and duration: } 0 \text { and } 60 \mathrm{mg} \text { of essential oil of TV and } \mathrm{NS} \mathrm{kg}^{-1} \text { of } \\
\text { diet and } 38 \mathrm{~d} \text { of supplementation } \\
\text { Husbandry conditions: } 5 \text { birds } \times \text { cage }(50 \times 60 \mathrm{~cm}), 8 \text { replicates per } \\
\text { treatment; temperature (N.A.), moisture (N.A.) and illumination ( } 24 \text { h); } \\
\text { vaccine (N.A.) } \\
\text { Slaughter: fasting time (N.A.) and method (cervical dislocation) } \\
\text { Sex: not sexed (Ø) }\end{array}$ & 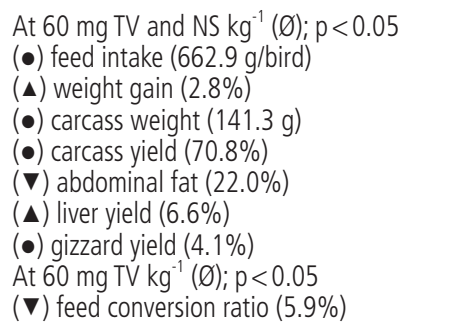 & Denli et al [47] \\
\hline Thyme (Thymus vulgaris L) & $\begin{array}{l}\text { Age and weight: } 7 \mathrm{~d} \text {-old/N.A. } \\
\text { Dose and duration: } 0 \text { and } 2.5 \mathrm{~mL} \text { of essential oil } \mathrm{kg}^{-1} \text { of diet and } 150 \mathrm{~d} \\
\text { of supplementation } \\
\text { Husbandry conditions: } 18 \text { birds } \times \text { cage (N.A.), } 3 \text { replicates per treatment; } \\
\text { temperature }\left(21.7^{\circ} \mathrm{C}\right) \text {, moisture (N.A.) and illumination ( } 24 \mathrm{~h} \text { during the } \\
\text { first wk and } 18 \mathrm{~h} \text { during the next steps of the experiment); vaccine (N.A.) } \\
\text { Slaughter: fasting time (N.A.) and method (N.A.) } \\
\text { Sex: female (O) and male ( }\left(^{-1}\right)\end{array}$ & $\begin{array}{l}\text { At } 0 \text { and } 2.5 \mathrm{~mL} \mathrm{~kg}^{-1}(\mathrm{O}) ; \mathrm{p}<0.05 \\
(\bullet) \text { live weight }(172.65 \mathrm{~g}) \\
(\bullet) \text { carcass weight }(106.7 \mathrm{~g}) \\
(\mathbf{)}) \text { feed intake }(1.8 \%) \\
(\bullet) \text { feed conversion ratio }(3.5) \\
\text { At } 0 \text { and } 2.5 \mathrm{~mL} \mathrm{~kg}\left(\mathrm{~d}^{-1}\right) ; \mathrm{p}<0.05 \\
(\bullet) \text { live weight }(150.55 \mathrm{~g}) \\
(\bullet) \text { carcass weight }(99.8 \mathrm{~g})\end{array}$ & Sengül et al [48] \\
\hline Duckweed (Wolffia globosa L.) & $\begin{array}{l}\text { Age and weight: } 7 \mathrm{~d}-\mathrm{old} / \mathrm{N} . \mathrm{A} . \\
\text { Dose and duration: } 0 \%, 25 \%, 50 \% \text {, and } 75 \% \text { powder and } 42 \mathrm{~d} \text { of } \\
\text { supplementation } \\
\text { Husbandry conditions: } 12 \text { birds } \times \text { cage (N.A.), } 6 \text { replicates per treatment; } \\
\text { temperature }\left(32^{\circ} \mathrm{C} \text { during } 2 \mathrm{wk} \text { and } 25^{\circ} \mathrm{C} \text { during the next steps of the }\right. \\
\text { experiment), moisture (N.A.) and illumination ( } 24 \mathrm{~h} \text { ); vaccine (N.A.) } \\
\text { Slaughter: fasting time (N.A.) and method (cervical dislocation) } \\
\text { Sex: not sexed (Ø) }\end{array}$ & $\begin{array}{l}\text { At } 25 \% \text { to } 75 \%(\varnothing) ; p<0.05 \\
(\nabla) \text { feed intake }(9.9 \%) \\
(\bullet) \text { feed conversion ratio }(3.7) \\
(\bullet) \text { carcass yield }(76.7 \%) \\
\text { (•) breast yield }(6.8 \%) \\
(\bullet) \text { leg yield }(17.5 \%) \\
(\bullet) \text { wing yield }(20.8 \%)\end{array}$ & $\begin{array}{c}\text { Chantiratikul et al } \\
\text { [49] }\end{array}$ \\
\hline Thyme (Thymus vulgaris) & $\begin{array}{l}\text { Age and weight: } 1 \mathrm{~d} \text {-old/N.A. } \\
\text { Dose and duration: } 0 \text { and } 1 \mathrm{~g} \text { of essential oil } \mathrm{kg}^{-1} \text { and } 35 \mathrm{~d} \text { of supple- } \\
\text { mentation } \\
\text { Husbandry conditions: } 15 \text { birds } \times \text { cage (N.A.), } 5 \text { replicates per treatment; } \\
\text { temperature }\left(32^{\circ} \mathrm{C} \text {, with a reduction of } 3^{\circ} \mathrm{C} \text { per wk), moisture (N.A.) and }\right. \\
\text { illumination }(24 \mathrm{~h}) \text {; vaccine (N.A.) } \\
\text { Slaughter: fasting time (N.A.) and method (N.A.) } \\
\text { Sex: male }\left({ }^{\prime}\right)\end{array}$ & $\begin{array}{l}\text { At } 1 \mathrm{~g} \mathrm{~kg}^{-1}\left(\mathrm{o}^{-1}\right) ; \mathrm{p}<0.05 \\
(\bullet) \text { feed intake }(450.5 \mathrm{~g} / \mathrm{bird}) \\
(\bullet) \text { feed conversion ratio }(2.8) \\
(\mathbf{\Delta}) \text { live weight }(7.1 \%) \\
(\mathbf{\Delta}) \text { carcass yield }(2.5 \%) \\
(\mathbf{\Delta}) \text { breast yield }(6.9 \%) \\
(\bullet) \text { leg yield }(15.4 \%) \\
(\bullet) \text { abdominal fat yield }(1.8 \%) \\
(\bullet) \text { liver yield }(3.3 \%) \\
(\bullet) \text { heart yield }(0.71 \%) \\
(\mathbf{v}) \text { CFU for E. coli }(10.7 \%) \\
(\mathbf{\Delta}) \text { CFU for Lactobacillus }(10.4 \%)\end{array}$ & Khaksar et al [50] \\
\hline $\begin{array}{l}\text { Peppermint } \\
\text { (Mentha spicata) }\end{array}$ & $\begin{array}{l}\text { Age and weight: } 7 \mathrm{~d} \text {-old } / 34.9 \mathrm{~g} \\
\text { Dose and duration: } 0 \%, 1 \%, 2 \%, 3 \% \text {, and } 4 \% \text { of dried leaves and } 35 \mathrm{~d} \\
\text { of supplementation } \\
\text { Husbandry conditions: } 15 \text { birds } \times \text { cage (N.A.), } 4 \text { replicates per treat- } \\
\text { ment; temperature }\left(35^{\circ} \mathrm{C} \text {, with a reduction of } 3^{\circ} \mathrm{C} \text { per wk), moisture (N.A.) }\right. \\
\text { and illumination (N.A.); vaccine (N.A.) } \\
\text { Slaughter: fasting time (N.A.) and method (cervical dislocation) } \\
\left.\text { Sex: male ( }{ }^{2}\right)\end{array}$ & 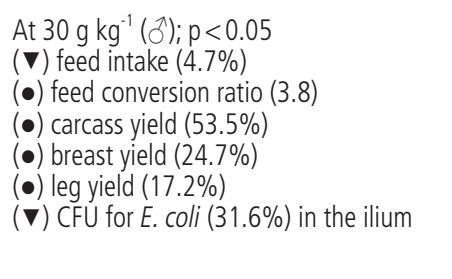 & $\begin{array}{c}\text { Ghazaghi et al } \\
{[51]}\end{array}$ \\
\hline $\begin{array}{l}\text { Peppermint } \\
\quad \text { (Mentha piperita) }\end{array}$ & $\begin{array}{l}\text { Age and weight: } 7 \mathrm{~d}-0 \mathrm{ld} / 29.6 \mathrm{~g} \\
\text { Dose and duration: } 0,10,20,30 \text {, and } 40 \mathrm{~g} \text { of dried leaves } \mathrm{kg}^{-1} \text { and } 35 \\
\mathrm{~d} \text { of supplementation } \\
\text { Husbandry conditions: } 12 \text { birds } \times \text { cage }(N . A .), 5 \text { replicates per treat- } \\
\text { ment; temperature }\left(35^{\circ} \mathrm{C} \text {, with a reduction of } 3^{\circ} \mathrm{C} \text { per wk), moisture }(60 \%)\right. \\
\text { and illumination (N.A.); vaccine (N.A.) } \\
\text { Slaughter: fasting time (N.A.) and method (cervical dislocation) } \\
\text { Sex: not sexed (Ø) }\end{array}$ & $\begin{array}{l}\text { At } 20 \text { to } 30 \mathrm{~g} \mathrm{~kg}^{-1}(\varnothing) ; p<0.05 \\
(\mathbf{\Delta}) \text { feed intake }(3.8 \%) ; p=0.008 \\
(\mathbf{\Delta}) \text { feed conversion ratio }(5.3 \%) ; p=0.029 \\
(\mathbf{\nabla}) \text { breast yield }(15.1 \%) ; p=0.047 \\
(\mathbf{v}) \text { leg yield }(12.5 \%) ; p=0.047 \\
(\bullet) \text { heart yield }(0.9 \%) \\
(\bullet) \text { liver yield }(2.5 \%) \\
(\mathbf{v}) \text { CFU for coliforms }(19.7 \%) ; p=0.001 \\
(\mathbf{\Delta}) \text { CFU for lactic acid bacteria }(24.3 \%) ; \\
p=0.001\end{array}$ & Mehri et al [52] \\
\hline
\end{tabular}

N.A., not available; TV, Thymus vulgaris L.; NS, Nigella sativa L.; VC, vitamin C; FA, folic acid; $(\mathbf{\Lambda})$, significant increase with respect to the control group; $(\boldsymbol{\nabla})$, significant reduction with respect to the control group; $(\bullet)$, without significant differences with respect to the control group; CFU, colony-forming unit. 
Table 2. Carcass characteristics of Japanese quail supplemented with natural ingredients in their diet (Continued)

\begin{tabular}{|c|c|c|c|}
\hline Natural ingredients & Bird management & Relevant results & References \\
\hline $\begin{array}{l}\text { Green tea } \\
\quad \text { (Camellia sinensis) }\end{array}$ & $\begin{array}{l}\text { Age and weight: } 4 \mathrm{~d} \text {-old/N.A. } \\
\text { Dose and duration: } 0,1.25 \text {, and } 2.5 \mathrm{~g} \text { of powdered leaves } \mathrm{kg}^{-1} \text { of diet and } 30 \mathrm{~d} \\
\text { of supplementation } \\
\text { Husbandry conditions: } 17 \text { birds } \times \text { cage }(45 \times 100 \mathrm{~cm}), 5 \text { replicates per treat- } \\
\text { ment; temperature }\left(21.7^{\circ} \mathrm{C}\right) \text {, moisture }(45 \%) \text { and illumination (N.A.); vaccine (N.A.) } \\
\text { Slaughter: fasting time (N.A.) and method (N.A.) } \\
\text { Sex: not sexed (Ø) }\end{array}$ & $\begin{array}{l}\text { At } 1.25 \text { to } 2.5 \mathrm{~g} \mathrm{~kg}^{-1}(\varnothing) ; \mathrm{p}>0.05 \\
(\bullet) \text { feed intake }(494 \mathrm{~g} / \mathrm{bird}) \\
(\bullet) \text { feed conversion ratio }(3.4) \\
(\bullet) \text { carcass yield }(66.4 \%) \\
(\bullet) \text { heart yield }(0.87 \%) \\
(\bullet) \text { liver yield }(2.4 \%) \\
(\bullet) \text { gizzard yield }(2.1 \%)\end{array}$ & Kara et al [53] \\
\hline
\end{tabular}

Plants

Fever tea (Lippia javanica)

Canola

(Brassica napus)
Age and weight: $8 \mathrm{~d}$-old/N.A.

Dose and duration: 0 and $25 \mathrm{~g}$ of powder $\mathrm{kg}^{-1}$ and $32 \mathrm{~d}$ of supplementation Husbandry conditions: 7 birds $\times$ cage (N.A.), 5 replicates per treatment; temperature (N.A.), moisture (N.A.) and illumination (N.A.); vaccine (N.A.) Slaughter: fasting time (13 h) and method (cervical dislocation) Sex: male ( $\left.{ }^{\lambda}\right)$

Age and weight: $150 \mathrm{~d}$-old/158.3 g

Dose and duration:
supplementation

Husbandry conditions: 7 birds $\times$ cage (N.A.), 4 replicates per treatment; temperature (N.A.), moisture (N.A.) and illumination (N.A.); vaccines (N.A.)

Slaughter: fasting time (13 h) and method (stunning with carbon dioxide and subsequent cervical dislocation)

Sex: female (ㅇ)
At $25 \mathrm{~g} \mathrm{~kg}^{-1}\left(\mathrm{O}^{-1}\right): p<0.05$

- feed intake (582 g/bird)

(•) feed conversion ratio (N.A.)

(•) hot carcass yield (114.3 g)

(•) cold carcass yield (76.2 g)

(v) gizzard weight $(27.5 \%)$

(•) heart weight $(1.3 \mathrm{~g})$

(•) liver weight (1.7 g)

At 0 to $17.5 \%$ (Q); $p<0.05$

(•) feed intake (220 g/bird)

(•) feed conversion ratio (N.A.)

(•) weight gain (71.2 g)

(•) gizzard weight (3.1 g)

(•) heart weight $(1.7 \mathrm{~g})$

(•) liver weight (4.2 g)

(•) hot carcass weight (135.2 g)

(•) cold carcass weight (133.0 g)

(•) wing length $(9.4 \mathrm{~cm})$

(•) leg length (3.8 c)

At 0 and $5.0 \%(+$ ) $; p<0.05$

$(\boldsymbol{\Delta})$ small intestine length (6.3\%)

At $100 \mathrm{mg} \mathrm{kg}^{-1}\left({ }^{3}\right) ; p<0.05$

(A) live weight (6.2\%); $p=0.017$

$(\boldsymbol{\nabla})$ feed conversion ratio $(6.6 \%) ; p=$ 0.003

Dose and duration: 0,100 , and $200 \mathrm{mg}$ of oil $\mathrm{kg}^{-1}$ and 1 and $2 \mathrm{~g}$ of powder $\mathrm{kg}-1$ and $35 \mathrm{~d}$ of supplementation

Husbandry conditions: 15 birds $\times$ cage $\left(100 \times 100 \mathrm{~cm}^{2}\right)$, 4 replicates per treatment; temperature $\left(37^{\circ} \mathrm{C}\right.$ to $\left.25^{\circ} \mathrm{C}\right)$, moisture (N.A.) and illumination (24 h, At $2 \mathrm{~g} \mathrm{~kg}^{-1}\left({ }^{-1}\right)$

fluorescent light of 20-|x intensity); vaccine (N.A.)

Slaughter: fasting time (12 h) and method (cervical dislocation) Sex: male (ふ)

( $\mathbf{\Delta})$ live weight $(1.7 \%) ; p=0.017$

( $\mathbf{\nabla})$ feed conversion ratio $(1.7 \%) ; p=$ 0.003

At $100 \mathrm{mg} \mathrm{kg}^{-1}$ and $2 \mathrm{~g} \mathrm{~kg}^{-1}\left(\mathrm{~J}^{1}\right) ; \mathrm{p}<0.05$

(•) feed intake $(538.4 \mathrm{~g} / \mathrm{bird}) ; p=0.802$
Mnisi et al [54]

Mnisi and Mlambo [55]

At $2 \%(\varnothing) ; p<0.05$

Age and weight: 3 d-old/N.A.

( $\mathbf{\nabla})$ feed conversion ratio $(10.1 \%)$

tation

$(\mathbf{v})$ feed conversion ratio
$(\mathbf{\Delta})$ weight gain $(6.3 \%)$

treatment (N.A.); temperature $\left(37^{\circ} \mathrm{C}\right.$ to $\left.25^{\circ} \mathrm{C}\right)$, moisture (N.A.) and illumination $(\mathbf{\Delta})$ carcass yield $(4.7 \%)$ (23 $\mathrm{h}$ during the first four wk and $14 \mathrm{~h}$ during the last two wk); vaccines (N.A.) ( $\mathbf{\nabla})$ abdominal fat (17.6\%)

Slaughter: fasting time (N.A.) and method (stunning with carbon dioxide and ( $(\mathbf{\Delta})$ liver yield (17.2\%) subsequent cervical dislocation)

$(\boldsymbol{\Delta})$ liver yield $(17.2 \%)$
$(\bullet)$ heart yield $(0.78 \%)$

Sex: not sexed ( $\varnothing)$

Age and weight: $1 \mathrm{~d}-\mathrm{old} / 9.9 \mathrm{~g}$

At $60 \%$ raw seeds $(\varnothing) ; p<0.05$

Chickpea

(Cicer arietinum L.)
Mehdipour et al

Güler et al [56]

Obregón et al [14]
Dose and duration: $0 \%, 60 \%$ of raw seeds, and $60 \%$ of cooked seeds and $35 \mathrm{~d}$ of supplementation

Husbandry conditions: 20 birds $\times$ cage $(90 \times 90 \times 60 \mathrm{~cm})$, replicates per treatment (N.A.); temperature $\left(35^{\circ} \mathrm{C}\right.$ to $38^{\circ} \mathrm{C}$ during the first four days, and a reduction of $5^{\circ} \mathrm{C}$ per wk during the next steps), moisture (67\%) and illumination (N.A.); vaccine (N.A.)

Slaughter: fasting time (N.A.) and method (cervical dislocation) Sex: not sexed (Ø)
( $\mathbf{\Delta})$ feed intake $(2.9 \%) ; p<0.01$

(•) feed conversion ratio (N.A.)

(ム) live weight $(7.5 \%) ; p<0.01$

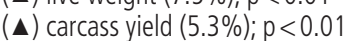

N.A., not available; TV, Thymus vulgaris L.; NS, Nigella sativa L.; VC, vitamin C; FA, folic acid; ( $\mathbf{\Lambda}$ ), significant increase with respect to the control group; ( $\mathbf{\nabla})$, significant reduction with respect to the control group; $(\bullet)$, without significant differences with respect to the control group; CFU, colony-forming unit. 
Table 2. Carcass characteristics of Japanese quail supplemented with natural ingredients in their diet (Continued)

\begin{tabular}{|c|c|c|c|}
\hline Natural ingredients & Bird management & Relevant results & References \\
\hline \multicolumn{4}{|l|}{ Worms } \\
\hline Earthworms (N.A.) & $\begin{array}{l}\text { Age and weight: } 42 \mathrm{~d} \text {-old/N.A. } \\
\text { Dose and duration: } 0 \% \text { and } 6 \% \text { powder and } 42 \mathrm{~d} \text { of supplementation } \\
\text { Husbandry conditions: } 5 \text { birds } \times \text { cage (N.A.), } 5 \text { replicates per treatment; tempera- } \\
\text { ture (N.A.), moisture (N.A.) and illumination (N.A.); vaccine (N.A.) } \\
\text { Slaughter: fasting time (N.A.) and method (N.A.) } \\
\text { Sex: not sexed (Ø) }\end{array}$ & 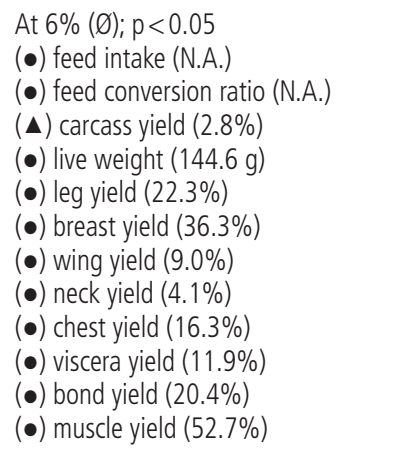 & $\begin{array}{c}\text { Morón-Fuenmayor et } \\
\text { al [13] }\end{array}$ \\
\hline $\begin{array}{l}\text { Earthworms } \\
\quad \text { (Eisenia foetida) }\end{array}$ & $\begin{array}{l}\text { Age and weight: } 1 \mathrm{~d}-0 \mathrm{dd} / 7.59 \mathrm{~g} \\
\text { Dose and duration: } 0 \% \text { and } 4 \% \text { powder and } 180 \mathrm{~d} \text { of supplementation } \\
\text { Husbandry conditions: } 3 \text { birds } \times \text { cage }(32 \times 50 \times 30 \mathrm{~cm}), 6 \text { replicates per treat- } \\
\text { ment; temperature }\left(22.5^{\circ} \mathrm{C}\right) \text {, moisture }(64 \%) \text { and illumination (N.A.); vaccines } \\
\text { (N.A.) } \\
\text { Slaughter: fasting time (N.A.) and method (N.A.) } \\
\text { Sex: without sexing (Ø) }\end{array}$ & $\begin{array}{l}\text { At } 4 \%(\varnothing) ; p<0.05 \\
(\bullet) \text { feed intake }(474.6 \mathrm{~g} / \mathrm{bird}) \\
(\bullet) \text { feed conversion ratio }(3.8) \\
(\mathbf{\Delta}) \text { weight gain }(8.2 \%) \\
(\mathbf{\Delta}) \text { carcass yield }(4.4 \%)\end{array}$ & Díaz-Cuellar et al [10] \\
\hline \multicolumn{4}{|l|}{ Bee products } \\
\hline Propolis (bee glue) & $\begin{array}{l}\text { Age and weight: } 1 \mathrm{~d}-\mathrm{old} / 9.2 \mathrm{~g} \\
\text { Dose and duration: } 0,0.5,1.0 \text {, and } 1.5 \mathrm{~g} \mathrm{~kg} \text { and } 35 \mathrm{~d} \text { of supplementation } \\
\text { Husbandry conditions: } 3 \text { birds } \times \text { cage }(30 \times 50 \mathrm{~cm}), 10 \text { replicates per treat- } \\
\text { ment; temperature (N.A.), moisture (N.A.) and illumination ( } 24 \mathrm{~h}) \text {; vaccine (N.A.) } \\
\text { Slaughter: fasting time (N.A.) and method (cervical dislocation) } \\
\text { Sex: not sexed (Ø) }\end{array}$ & $\begin{array}{l}\text { At } 0.5 \text { to } 1.5 \mathrm{~g} \mathrm{~kg}^{-1}(\varnothing) ; p<0.05 \\
(\bullet) \text { feed intake }(625.6 \mathrm{~g} / \mathrm{bird}) \\
(\bullet) \text { feed conversion ratio (N.A.) } \\
(\mathbf{\Delta}) \text { weight gain }(6.9 \%) ; p<0.01 \\
\text { At } 1.0 \text { to } 1.5 \mathrm{~g} \mathrm{~kg}^{-1} \\
(\mathbf{\Delta}) \text { carcass weight }(8.2 \%) ; p<0.01 \\
\text { At } 0 \text { to } 1.5 \mathrm{~g} \mathrm{~kg}-1 ; \mathrm{p}<0.05 \\
(\bullet) \text { carcass yield }(75.4 \%) \\
(\bullet) \text { abdominal fat weight }(1.2 \mathrm{~g}) \\
(\bullet) \text { liver weight }(3.2 \mathrm{~g}) \\
(\bullet) \text { gizzard weight }(3.7 \mathrm{~g})\end{array}$ & Denli et al [57] \\
\hline Propolis (bee glue) & $\begin{array}{l}\text { Age and weight: } 8 \mathrm{~d} \text {-old } / 9.12 \mathrm{~g} \\
\text { Dose and duration: } 0 \%, 0.5 \% \text {, and } 1 \% \text { of ethanolic extract and } 35 \mathrm{~d} \text { of sup- } \\
\text { plementation } \\
\text { Husbandry conditions: } 3 \text { birds } \times \text { cage }(50 \times 50 \times 17 \mathrm{~cm}), 3 \text { replicates per treat- } \\
\text { ment; temperature (N.A.), moisture (N.A.) and illumination ( } 24 \text { h); vaccines (N.A.) } \\
\text { Slaughter: fasting time (N.A.) and method (N.A.) } \\
\text { Sex: not sexed (Ø) }\end{array}$ & $\begin{array}{l}\text { At } 1 \%(\varnothing) ; p<0.05 \\
(\bullet) \text { feed intake }(615.7 \mathrm{~g} / \mathrm{bird}) \\
(\bullet) \text { feed conversion ratio }(2.7) \\
(\bullet) \text { weight gain }(>2 \%) \text { a } 21 \mathrm{~d} \\
\text { At } 0 \text { and } 1 \% ; p<0.05 \\
(\bullet) \text { weight gain }(224.3 \mathrm{~g}) \\
(\bullet) \text { live weight }(246.3 \mathrm{~g}) \\
(\bullet) \text { carcass weight }(181.7 \mathrm{~g}) \\
(\bullet) \text { carcass yield }(73.7 \%) \\
(\bullet) \text { liver yield }(2.7 \%) \\
(\bullet) \text { heart yield }(1.2 \%) \\
(\bullet) \text { gizzard yield }(3.0 \%)\end{array}$ & Canogullari et al [58] \\
\hline Pollen & $\begin{array}{l}\text { Age and weight: } 8 \mathrm{~d}-0 \mathrm{dd} / 8.29 \mathrm{~g} \\
\text { Dose and duration: } 0,5,10, \text { and } 20 \mathrm{~g} \text { powder } \mathrm{kg}^{-1} \text { and } 35 \mathrm{~d} \text { of supplementa- } \\
\text { tion } \\
\text { Husbandry conditions: } 3 \text { birds } \times \text { cage }(50 \times 50 \times 17 \mathrm{~cm}), 3 \text { replicates per treat- } \\
\text { ment; temperature (N.A.), moisture (N.A.) and illumination ( } 24 \mathrm{~h}) \text {; vaccines (N.A.) } \\
\text { Slaughter: fasting time (N.A.) and method (N.A.) } \\
\text { Sex: not sexed (Ø) }\end{array}$ & $\begin{array}{l}\text { At } 5 \text { to } 20 \mathrm{~g} \mathrm{~kg}^{-1}(\varnothing) ; p<0.05 \\
(\mathbf{\Delta}) \text { feed intake }(6.9 \%) \\
(\bullet) \text { feed conversion ratio }(2.7) \\
(\mathbf{\Delta}) \text { weight gain }(>2 \%) \text { a } 28 \mathrm{~d} \\
\text { At } 0 \text { to } 20 \mathrm{~g} \mathrm{~kg}^{-1} ; \mathrm{p}<0.05 \\
(\bullet) \text { weight gain }(223.3 \mathrm{~g}) \\
(\bullet) \text { live weight }(237.5 \mathrm{~g}) \\
(\bullet) \text { carcass weight }(177.3 \mathrm{~g}) \\
(\bullet) \text { carcass yield }(74.6 \%) \\
(\bullet) \text { liver yield }(3.3 \%) \\
(\bullet) \text { heart yield }(1.3 \%) \\
(\bullet) \text { gizzard yield }(3.3 \%)\end{array}$ & \\
\hline
\end{tabular}

N.A., not available; TV, Thymus vulgaris L.; NS, Nigella sativa L.; VC, vitamin C; FA, folic acid; ( $\mathbf{\Delta})$, significant increase with respect to the control group; ( $\mathbf{\nabla})$, significant reduction with respect to the control group; $(\bullet)$, without significant differences with respect to the control group; CFU, colony-forming unit. 
Table 2. Carcass characteristics of Japanese quail supplemented with natural ingredients in their diet (Continued)

\begin{tabular}{|c|c|c|c|}
\hline Natural ingredients & Bird management & Relevant results & References \\
\hline \multicolumn{4}{|l|}{ Chemical compounds } \\
\hline Vitamin C and folic acid & $\begin{array}{l}\text { Age and weight: } 10 \mathrm{~d} \text {-old } / 46.4 \mathrm{~g} \\
\text { Dose and duration: } 0,250 \mathrm{mg} \mathrm{VC,} 1 \mathrm{mg} \mathrm{FA} \text {, and } 250 \mathrm{mg} \text { VC+1 mg FA (VC+FA) } \\
\text { and } 42 \mathrm{~d} \text { of supplementation } \\
\text { Husbandry conditions: } 10 \text { birds } \times \text { cage (N.A.), } 3 \text { replicates per treatment; tempera- } \\
\text { ture }\left(18^{\circ} \mathrm{C} \text { to } 22^{\circ} \mathrm{C}\right) \text {, moisture (N.A.) and illumination (N.A.); vaccine (N.A.) } \\
\text { Slaughter: fasting time (N.A.) and method (N.A.) } \\
\text { Sex: not sexed (Ø) }\end{array}$ & $\begin{array}{l}\text { At VC+FA (Ø); } p<0.05 \\
(\mathbf{\nabla}) \text { feed intake }(1.8 \%) \\
(\bullet) \text { feed conversion ratio (N.A.) } \\
(\mathbf{v}) \text { live weight }(4 \%) \\
(\mathbf{\nabla}) \text { carcass weight }(15.3 \%) \\
(\mathbf{\nabla}) \text { carcass yield }(1.3 \%)\end{array}$ & Sahin et al [59] \\
\hline Lycopene & $\begin{array}{l}\text { Age and weight: } 10 \mathrm{~d} \text {-old } / 33.3 \mathrm{~g} \\
\text { Dose and duration: } 0,50,100 \text {, and } 200 \mathrm{mg} \text { of oil } \mathrm{kg}^{-1} \text { and } 45 \mathrm{~d} \text { of supplemen- } \\
\text { tation } \\
\text { Husbandry conditions: } 3 \text { birds } \times \text { cage (N.A.), } 10 \text { replicates per treatment; } \\
\text { temperature }\left(22^{\circ} \mathrm{C}\right) \text {, moisture }(57 \%) \text { and illumination (N.A.); vaccine (N.A.) } \\
\text { Slaughter: fasting time ( } 24 \mathrm{~h}) \text { and method (N.A.) } \\
\text { Sex: not sexed }(\varnothing)\end{array}$ & $\begin{array}{l}\text { At } 200 \mathrm{mg} \mathrm{kg}^{-1}(\varnothing) ; p<0.05 \\
(\bullet) \text { feed intake }(658.3) \\
(\bullet) \text { feed conversion ratio (N.A.) } \\
(\boldsymbol{\Delta}) \text { weight gain }(2.0 \%) ; p=0.01 \\
(\mathbf{\Delta}) \text { carcass weight }(0.7 \%) ; p=0.01 \\
(\mathbf{\Delta}) \text { carcass yield }(0.3 \%) ; p=0.01\end{array}$ & Sahin et al [60] \\
\hline \multicolumn{4}{|l|}{ Mushroom } \\
\hline $\begin{array}{l}\text { Common mushroom } \\
\text { (Agaricus bisporus) }\end{array}$ & $\begin{array}{l}\text { Age and weight: } 7 \mathrm{~d}-\mathrm{old} / \mathrm{N} . \mathrm{A} \text {. } \\
\text { Dose and duration: } 0 \%, 0.5 \%, 1.0 \% \text {, and } 2.0 \% \text { powder and } 35 \mathrm{~d} \text { of supple- } \\
\text { mentation } \\
\text { Husbandry conditions: } 20 \text { birds } \times \text { cage }(100 \times 100 \mathrm{~cm}), 3 \text { replicates per treat- } \\
\text { ment; temperature }\left(37^{\circ} \mathrm{C} \text {, with a reduction of } 3^{\circ} \mathrm{C} \text { per wk), moisture (N.A.), and }\right. \\
\text { illumination ( } 24 \mathrm{~h}) \text {; vaccine (N.A.) } \\
\text { Slaughter: fasting time (N.A.) and method (cervical dislocation) } \\
\left.\text { Sex: male ( }{ }^{\prime}\right)\end{array}$ & $\begin{array}{l}\text { At } 2 \%(ठ) ; p<0.05 \\
(\mathbf{\Delta}) \text { feed intake }(6 \%) ; p=0.005 \\
(\mathbf{\nabla}) \text { feed conversion ratio }(6.1 \%) ; p \\
=0.001 \\
(\mathbf{\Delta}) \text { weight gain }(12.6 \%) ; p<0.001 \\
(\mathbf{\Delta}) \text { liver yield }(16.4 \%) ; p<0.001 \\
(\bullet) \text { breast yield }(27.6 \%) \\
(\bullet) \text { leg yield }(20.5 \% \\
(\bullet) \text { heart yield }(0.84 \% \\
(\bullet) \text { intestine length }(61.2 \mathrm{~cm})\end{array}$ & Asadi-Dizaji et al [61] \\
\hline
\end{tabular}

N.A., not available; TV, Thymus vulgaris L.; NS, Nigella sativa L.; VC, vitamin C; FA, folic acid; ( $\mathbf{\Lambda}$ ), significant increase with respect to the control group; ( $\mathbf{\nabla})$, significant reduction with respect to the control group; $(\bullet)$, without significant differences with respect to the control group; CFU, colony-forming unit.

confirmed by research, including the effects of medicinal herbs (thyme, black seed, green tea, duckweed, and mint), plants (verbena and canola), spices (cinnamon), seeds (coriander and chickpea), worms (earthworm), apicultural products (propolis and pollen), chemical compounds (vitamin C, folic acid, and lycopene), and edible fungi (common mushroom).

Table 3 lists the effects of natural additives in the quail diet on meat quality, as confirmed by research, notably the improvement of meat quality through increased oxidative stability. The tested natural ingredients include medicinal herbs (spearmint and green tea), spices (cinnamon and laurel), vegetables (tomato), plants (verbena and canola), seeds (marijuana), insects (black soldier fly), and edible fungi (oyster mushroom).

These additives of natural origin are of interest to the food industry because of their antimicrobial and antioxidant properties, which are commonly associated with the presence of phenolic compounds, such as phenolic acids and flavonoids $[42,44]$.

\section{ABSORPTION AND METABOLISM OF PHENOLIC COMPOUNDS}

Natural ingredients provide a wide range of antimicrobial and antioxidant compounds, such as phenolic compounds, which, in the present case, can modify the intestinal microflora of birds, improving animal health and reinforcing the endogenous antioxidant system responsible for mitigating the effects of free radicals formed during the oxidative stress process [ 42 , $44,65,66]$. Phenolic compounds are widely distributed in nature and esterify with glucose or other carbohydrates (glycosides) or are present in the form of free aglycones. The antibacterial and antioxidant properties of these compounds in the bird will depend on their bioaccessibility and bioavailability as well as their absorption under normal physiological conditions following release from the food matrix into the mucosa of the intestine $[65,66]$. Dietary antioxidants that are non-bioavailable may pass through the intestine without being absorbed and reach the colon. At this point, they may ferment by the action of bacterial enzymes and then contribute toward creating an antioxidant environment by eliminating free radicals, counteracting the pro-oxidant effects of the diet $[66,67]$.

The intake of phenolic compounds in the diet may have adverse metabolic effects associated with the lower efficiency of nutrients, particularly proteins (decreased amino acid digestibility), and with the inhibition of digestive enzymes due to the interaction of their hydroxyl groups with the carbonyl groups of proteins $[68,69]$. Nevertheless, Brenes et al $[70]$ investigated the effect of including grape seed extract $(0.6,1.8$, and $3.6 \mathrm{~g} / \mathrm{kg}$ ) in a diet for broilers during $42 \mathrm{~d}$ on productive parameters (weight gain and feed intake), weight of internal 
Table 3. Meat quality of Japanese quail supplemented with natural ingredients in their diet

\begin{tabular}{|c|c|c|c|}
\hline Natural source & Management of birds & Relevant results & References \\
\hline \multicolumn{4}{|l|}{ Medicinal herbs } \\
\hline $\begin{array}{l}\text { Green tea } \\
\text { (Camellia sinensis) }\end{array}$ & $\begin{array}{l}\text { Age and weight: } 4 \mathrm{~d} \text {-old/N.A. } \\
\text { Dose and duration: } 0,1.25 \text {, and } 2.5 \mathrm{~g} \text { of powdered leaves } \mathrm{kg}^{-1} \text { and } 30 \\
\text { d of supplementation } \\
\text { Husbandry conditions: } 17 \text { birds } \times \text { cage }(45 \times 100 \mathrm{~cm}), 5 \text { replicates per } \\
\text { treatment; temperature }\left(21.7^{\circ} \mathrm{C}\right) \text {, moisture }(45 \%) \text { and illumination (N.A.); } \\
\text { vaccines (N.A) } \\
\text { Slaughter: fasting time (N.A.) and method (N.A.) } \\
\text { Sex: not sexed (Ø) } \\
\text { Meat/temperature and storage time: breast and leg/ }-20^{\circ} \mathrm{C} \text { until analysis, } \\
\text { storage time not specified }\end{array}$ & $\begin{array}{l}\text { At } 2.5 \mathrm{~g} \mathrm{~kg}^{-1} \text {; breast }(\varnothing) ; p=0.044 \\
(\mathbf{\Delta}) \text { water holding capacity }(5.4 \%) \\
\text { At } 2.5 \mathrm{~g} \mathrm{~kg}^{-1} \text {; leg (Ø) } \\
(\bullet) \text { water holding capacity }(65.8 \%) ; \\
p<0.05\end{array}$ & Kara et al [53] \\
\hline $\begin{array}{l}\text { Peppermint } \\
\text { (Mentha piperita) }\end{array}$ & $\begin{array}{l}\text { Age and weight: } 8 \mathrm{~d} \text {-old } / 29.6 \mathrm{~g} \\
\text { Dose and duration: } 0,10,20,30 \text {, and } 40 \mathrm{~g} \text { of powdered leaves } \mathrm{kg}^{-1} \text { and } \\
7 \text { to } 35 \mathrm{~d} \text { of supplementation } \\
\text { Husbandry conditions: } 12 \text { birds } \times \text { cage (N.A.), } 5 \text { replicates per treat- } \\
\text { ment; temperature }\left(35^{\circ} \mathrm{C} \text { during the first wk, with a subsequent reduction }\right. \\
\text { of } 3^{\circ} \mathrm{C} \text { per wk), moisture }(60 \%) \text { and illumination (N.A.); vaccine (NewCas- } \\
\text { tle, only to measure humoral response) } \\
\text { Slaughter: fasting time (N.A.) and method (cervical dislocation) } \\
\text { Sex: not sexed (Ø) } \\
\text { Meat/temperature and storage time: leg } /-20^{\circ} \mathrm{C}, 30 \mathrm{~d}\end{array}$ & $\begin{array}{l}\text { At } 20 \text { to } 30 \mathrm{~g} \mathrm{~kg}^{-1}(\varnothing) \\
(\boldsymbol{\nabla}) \text { lipid oxidation-MDA }(65.0 \%) ; p= \\
0.001 \\
(\boldsymbol{\nabla}) \text { cooking loss }(14.75 \%) ; p<0.001 \\
\text { ( } \mathbf{v}) \text { drip loss }(2.7 \%) ; p<0.001 \\
(\bullet) \text { water holding capacity }(70.1 \%) \\
(\bullet) \text { pH value (6.4) }\end{array}$ & Mehri et al [62] \\
\hline $\begin{array}{l}\text { Peppermint } \\
\text { (Mentha spicata) }\end{array}$ & $\begin{array}{l}\text { Age and weight: } 7 \mathrm{~d}-0 \mathrm{ld} / 34.9 \mathrm{~g} \\
\text { Dose and duration: } 0 \%, 1 \%, 2 \%, 3 \% \text {, and } 4 \% \text { of powdered leaves and } \\
7 \text { to } 35 \mathrm{~d} \text { of supplementation } \\
\text { Husbandry conditions: } 15 \text { birds } \times \text { cage (N.A.), } 4 \text { replicates per treat- } \\
\text { ment; temperature }\left(35^{\circ} \mathrm{C} \text { during the first wk, with a subsequent reduction }\right. \\
\text { of } 3^{\circ} \mathrm{C} \text { per wk), moisture }(60 \%) \text { and illumination (N.A.); vaccine (N.A.) } \\
\text { Slaughter: fasting time (N.A.) and method (cervical dislocation) } \\
\text { Sex: not sexed (Ø) } \\
\text { Meat/temperature and storage time: breast and leg/ }-20^{\circ} \mathrm{C}, 90 \mathrm{~d}\end{array}$ & $\begin{array}{l}\text { At } 1 \% \text {; breast }(\varnothing) ; p<0.05 \\
\text { ( } \mathbf{\nabla}) \text { lipid oxidation-MDA }(17.5 \%) ; p<0.05 \\
\text { At } 1 \% \text {; leg }(\varnothing) \\
\text { ( } \mathbf{v}) \text { lipid oxidation-MDA }(16.5 \%) ; p<0.05\end{array}$ & Ghazaghi et al [51] \\
\hline \multicolumn{4}{|l|}{ Spices } \\
\hline $\begin{array}{l}\text { Cinnamon } \\
\quad \text { (Cinnamomum verum) }\end{array}$ & $\begin{array}{l}\text { Age and weight: } 420 \mathrm{~d} \text {-old/N.A. } \\
\text { Dose and duration: } 0,100 \text {, and } 200 \mathrm{mg} \text { of oil } \mathrm{kg}^{-1} \text { and } 1 \text { and } 2 \mathrm{~g} \text { of } \\
\text { powder } \mathrm{kg}^{-1} \text { and } 35 \mathrm{~d} \text { of supplementation } \\
\text { Husbandry conditions: } 15 \text { birds } \times \text { cage }\left(100 \times 100 \mathrm{~cm}^{2}\right) \text {, } 4 \text { replicates per } \\
\text { treatment; temperature }\left(37^{\circ} \mathrm{C} \text { to } 25^{\circ} \mathrm{C}\right) \text {, moisture (N.A.) and illumination } \\
(24 \mathrm{~h} \text {, fluorescent light of } 20-\mathrm{lx} \text { intensity); vaccine (N.A.) } \\
\text { Slaughter: fasting time }(12 \mathrm{~h}) \text { and method (cervical dislocation) } \\
\text { Sex: male ( }\left(^{-}\right) \\
\text {Meat/temperature and storage time: leg/ }-23^{\circ} \mathrm{C} \text { until analysis, stor- } \\
\text { age time not specified }\end{array}$ & 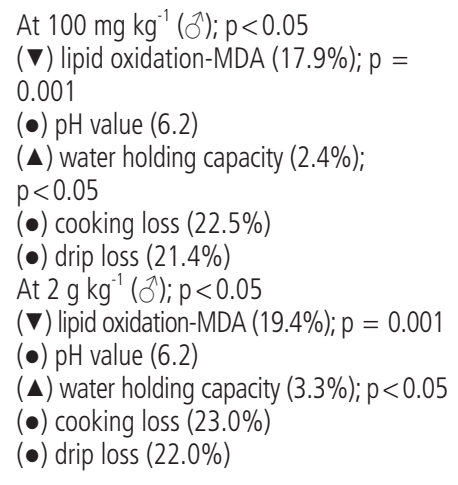 & Mehdipour et al [27] \\
\hline $\begin{array}{l}\text { Laurel } \\
\qquad \text { (Syzygium polyanthum) }\end{array}$ & $\begin{array}{l}\text { Age and weight: } 32 \mathrm{~d}-0 \mathrm{dd} / 101.94 \mathrm{~g} \\
\text { Dose and duration: } 0 \%, 1 \%, 2 \%, 3 \% \text {, and } 4 \% \text { powdered leaves and } \\
30 \mathrm{~d} \text { of supplementation } \\
\text { Husbandry conditions: } 5 \text { birds } \times \text { cage (N.A.), } 4 \text { replicates per treatment; } \\
\text { temperature (N.A.), moisture (N.A.) and illumination (N.A.); vaccine (N.A.) } \\
\text { Slaughter: fasting time (N.A.) and method (N.A.) } \\
\text { Sex: female (\$) } \\
\text { Meat/temperature and storage time: breast+leg/(N.A.) }{ }^{\circ} \mathrm{C} \text { (N.A.) }\end{array}$ & $\begin{array}{l}\text { At } 1 \% \text { to } 4 \% \text { N.A.; } p<0.05 \\
(\bullet) \text { fat content reduction }(5.5 \%)\end{array}$ & Adriani et al [63] \\
\hline
\end{tabular}

N.A., not available; $(\mathbf{\Lambda})$, significant increase with respect to the control group $(\mathbf{\nabla})$, significant reduction with respect to the control group; $(\bullet)$, without significant differences with respect to the control group; MDA, malondialdehyde.

organs (pancreas, spleen, and liver), length of digestive organs (duodenum, jejunum, ileum, and caecum), digestibility of phenolic compounds extracted from excreta, and antioxidant activity of the diet and excretions. The results showed that grape seed extract did not affect weight gain $(2.2 \mathrm{~kg})$, feed intake $(3.9 \mathrm{~kg})$, or the weight of the pancreas, spleen, or liver $(0.16 \%, 0.11 \%$, and $2 \%$, respectively). In the digestive organs, an increase was observed in the length of the duodenum and 
Table 3. Meat quality of Japanese quail supplemented with natural ingredients in their diet (Continued)

\begin{tabular}{|c|c|c|c|}
\hline Natural source & Management of birds & Relevant results & References \\
\hline \multicolumn{4}{|l|}{ Vegetables } \\
\hline $\begin{array}{l}\text { Tomato } \\
\text { (Lycopersicum esculentum) }\end{array}$ & $\begin{array}{l}\text { Age and weight: } 21 \mathrm{~d}-0 \text { ld/N.A. } \\
\text { Dose and duration: } 0 \%, 5 \% \text {, and } 10 \% \text { pulp and } 42 \mathrm{~d} \text { of supplementa- } \\
\text { tion } \\
\text { Husbandry conditions: } 6 \text { birds } \times \text { cage (N.A.), } 5 \text { replicates per treat- } \\
\text { ment; temperature (N.A.), moisture (N.A.) and illumination (N.A.); vaccine } \\
\text { (N.A.) } \\
\text { Slaughter: fasting time (N.A.) and method (N.A.) } \\
\text { Sex: not sexed (Ø) } \\
\text { Meat/temperature and storage time: raw and cooked breast } / 4^{\circ} \mathrm{C}, 9 \mathrm{~d}\end{array}$ & $\begin{array}{l}\text { At } 5 \% \text {; raw and cooked breast }(\varnothing) \text {; } \\
\text { p }<0.05 \\
(\mathbf{\nabla}) \text { lipid oxidation-MDA }(>10 \%)\end{array}$ & Botsoglou et al [25] \\
\hline \multicolumn{4}{|l|}{ Plants } \\
\hline Fever tea (Lippia javanica) & $\begin{array}{l}\text { Age and weight: } 8 \mathrm{~d} \text {-old/N.A. } \\
\text { Dose and duration: } 0 \text { and } 25 \mathrm{~g} \text { of powder } \mathrm{kg}^{-1} \text { and } 32 \mathrm{~d} \text { of supplemen- } \\
\text { tation } \\
\text { Husbandry conditions: } 7 \text { birds } \times \text { cage (N.A.), } 5 \text { replicates per treat- } \\
\text { ment; temperature (N.A.), moisture (N.A.) and illumination (N.A.); vaccine } \\
\text { (N.A.) } \\
\text { Slaughter: fasting time ( } 13 \mathrm{~h} \text { ) and method (cervical dislocation) } \\
\text { Sex: male ( }{ }^{\text {) }} \text { ) } \\
\text { Meat/temperature and storage time: raw and cooked breast } / 4^{\circ} \mathrm{C} \text {, } \\
24 \mathrm{~h} \text { postmortem }\end{array}$ & $\begin{array}{l}\text { At } 25 \mathrm{~g} \mathrm{~kg}^{-1} \text {; raw breast }\left(\jmath^{1}\right) ; p<0.05 \\
(\bullet) \mathrm{pH} \text { value }(6.9) \\
(\bullet) \mathrm{L}^{*} \text { value }(46.3) \\
(\bullet) \mathrm{a}^{*} \text { value }(3.2) \\
(\mathbf{\Delta}) \mathrm{b}^{*} \text { value }(53.5) \\
(\bullet) \text { C value }(5.8) \\
(\bullet) \text { h value }(1.4) \\
(\mathbf{\nabla}) \text { cooking loss }(16.5 \%)\end{array}$ & Mnisi et al [54] \\
\hline $\begin{array}{l}\text { Canola } \\
\text { (Brassica napus) }\end{array}$ & $\begin{array}{l}\text { Age and weight: } 150 \mathrm{~d} \text {-old } / 158.3 \mathrm{~g} \\
\text { Dose and duration: } 0 \%, 2.5 \%, 5.0 \%, 12.5 \% \text {, and } 17.5 \% \text { powder and } \\
150 \mathrm{~d} \text { of supplementation } \\
\text { Husbandry conditions: } 7 \text { birds } \times \text { cage (N.A.), } 4 \text { replicates per treat- } \\
\text { ment; temperature (N.A.), moisture (N.A.) and illumination (N.A.); vaccine } \\
\text { (N.A.) } \\
\text { Slaughter: fasting time ( } 13 \mathrm{~h} \text { ) and method (stunning with carbon diox- } \\
\text { ide and subsequent cervical dislocation) } \\
\text { Sex: female (Q) } \\
\text { Meat/temperature and storage time: breast } / 2^{\circ} \mathrm{C}, 24 \mathrm{~h} \text { post mortem }\end{array}$ & $\begin{array}{l}\text { At } 12.5 \% \text { and } 17.5 \%(\phi) ; p<0.05 \\
(\mathbf{v}) C^{*} \text { value }(31.5 \%) \\
\text { At } 0 \% \text { and } 17.5 \% ;(\text {; }) \\
(\bullet) \text { temperature }\left(18.4^{\circ} \mathrm{C}\right) \\
(\bullet) L^{*} \text { value }(49.5) \\
(\bullet) a^{*} \text { value }(2.3) \\
(\bullet) b^{*} \text { value }(4.7) \\
(\bullet) h^{*} \text { value }(1.1)\end{array}$ & $\begin{array}{l}\text { Mnisi and Mlambo } \\
\text { [55] }\end{array}$ \\
\hline \multicolumn{4}{|l|}{ Seeds } \\
\hline $\begin{array}{l}\text { Marijuana } \\
\text { (Cannabis sativa L.) }\end{array}$ & 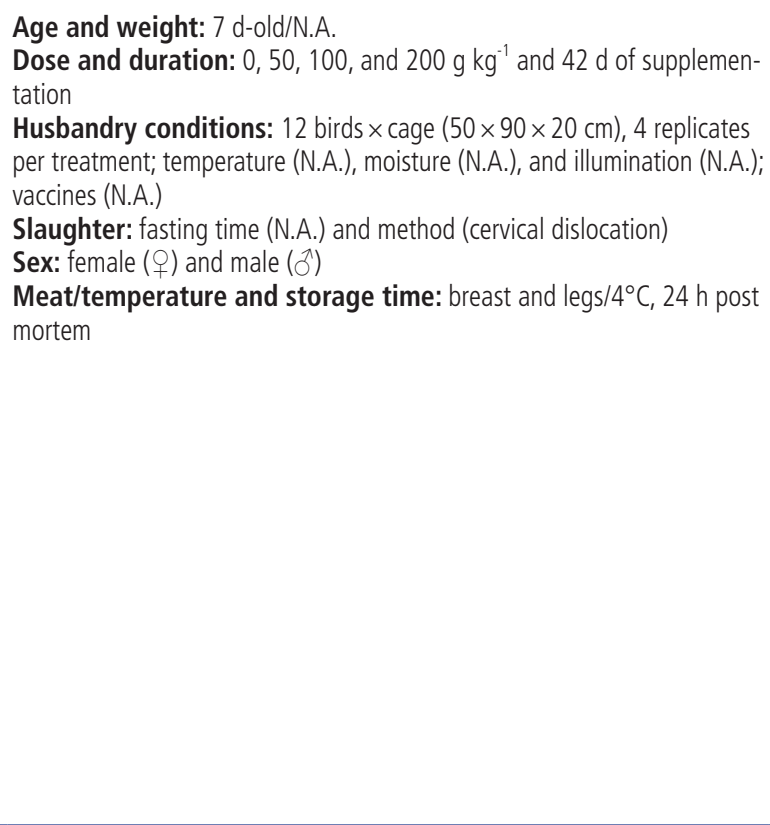 & 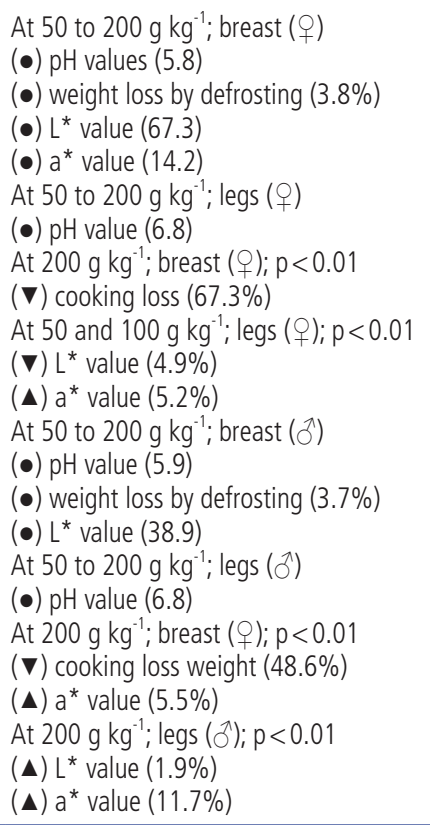 & Yalcin et al [64] \\
\hline
\end{tabular}

N.A., not available; $(\mathbf{\Lambda})$, significant increase with respect to the control group $(\mathbf{v})$, significant reduction with respect to the control group; $(\bullet)$, without significant differences with respect to the control group; MDA, malondialdehyde. 
Table 3. Meat quality of Japanese quail supplemented with natural ingredients in their diet (Continued)

\begin{tabular}{|c|c|c|c|}
\hline Natural source & Management of birds & Relevant results & References \\
\hline \multicolumn{4}{|l|}{ Insects } \\
\hline $\begin{array}{l}\text { Black soldier fly } \\
\text { (Hermetia illucens) }\end{array}$ & $\begin{array}{l}\text { Age and weight: } 10 \mathrm{~d}-0 \mathrm{ld} / \mathrm{N} . \mathrm{A} \text {. } \\
\text { Dose and duration: } 0 \%, 10 \% \text {, and } 15 \% \text { larvae and } 28 \mathrm{~d} \text { of } \\
\text { supplementation } \\
\text { Husbandry conditions: } 130 \text { birds } \times \text { cage (N.A.), } 5 \text { replicates per } \\
\text { treatment; temperature (N.A.), moisture (N.A.), and illumination } \\
\text { (N.A.); vaccine (N.A.) } \\
\text { Slaughter: fasting time }(6 \mathrm{~h} \text { ) and method (previous electrical } \\
\text { stunning and posterior cervical dislocation) } \\
\text { Sex: not sexed (Ø) } \\
\text { Meat/temperature and storage time: breast } /-40^{\circ} \mathrm{C}, 2 \mathrm{wk}\end{array}$ & $\begin{array}{l}\text { At } 0 \% \text { to } 15 \% \text {; }(\varnothing) \\
(\bullet) \text { moisture }(75.4 \%) \\
\text { (•) protein }(18.5 \%) \\
(\bullet) \text { fat }(4.6 \%) \\
(\bullet) \text { ash }(1.6 \%) \\
(\bullet) \text { lipid oxidation-MDA }\left(0.36 \mathrm{mg} \mathrm{MDA} \mathrm{kg}^{-1}\right)\end{array}$ & Cullere et al [21] \\
\hline \multicolumn{4}{|l|}{ Mushroom } \\
\hline $\begin{array}{l}\text { Oyster mushroom } \\
\quad \text { (Pleurotus ostreatus) }\end{array}$ & $\begin{array}{l}\text { Age and weight: } 1 \mathrm{~d}-\mathrm{old} / 14.54 \mathrm{~g} \\
\text { Dose and duration: } 0,10 \text {, and } 10 \mathrm{~g} \text { of powder } \mathrm{kg}^{-1} \text { and } 35 \mathrm{~d} \text { of } \\
\text { supplementation } \\
\text { Husbandry conditions: } 12 \text { birds } \times \text { cage }(90 \times 90 \times 60 \mathrm{~cm}), 8 \\
\text { replicates per treatment; temperature }\left(21^{\circ} \mathrm{C} \text { to } 36^{\circ} \mathrm{C}\right) \text {, moisture } \\
(23.4 \% \text { to } 53.8 \%) \text {, and illumination }(24 \mathrm{~h}) \text {; vaccine (without) } \\
\text { Slaughter: fasting time }(3 \mathrm{~h}) \text { and method (cervical dislocation) } \\
\left.\text { Sex: male ( }{ }^{\lambda}\right) \\
\text { Meat/temperature and storage time: breast } / 4^{\circ} \mathrm{C}, 15 \text { days }\end{array}$ & $\begin{array}{l}\text { At } 0 \text { to } 20 \mathrm{~g} \mathrm{~kg}^{-1} ; \text { carcass }\left({ }^{\Uparrow}\right) ; p<0.05 \\
(\bullet) \text { carcass weight }(133.0 \mathrm{~g}) \\
(\bullet) \text { carcass yield }(61.3 \%) \\
\text { At } 10 \text { and } 20 \mathrm{~g} \mathrm{~kg}^{-1} ; \text { breast }\left(\mathrm{o}^{-1}\right) \\
(\bullet) \text { moisture }(72.7 \%) \\
(\bullet) \text { protein }(22.2 \%) \\
(\bullet) \text { ash }(1.4 \%) \\
(\mathbf{\nabla}) \text { fat }(23.9 \%) ; p<0.001 \\
(\mathbf{\nabla}) L^{*} \text { value }(8.4 \%) ; p=0.011 \\
(\mathbf{\Delta}) a^{*} \text { value }(26.9 \%) ; p<0.001 \\
(\mathbf{\nabla}) b^{*} \text { value }(12.2 \%) ; p<0.001 \\
(\mathbf{\Delta}) \text { water holding capacity }(7.2 \%) ; p=0.017 \\
(\mathbf{\nabla}) \text { cooking loss weight }(26.8 \%) ; p=0.017 \\
(\mathbf{\nabla}) \text { hardness }(30 \%) ; p=0.037 \\
(\mathbf{\nabla}) \text { lipid oxidation-MDA }(33.5 \%) ; p<0.001\end{array}$ & $\begin{array}{c}\text { Vargas-Sánchez et al } \\
{[37]}\end{array}$ \\
\hline
\end{tabular}

N.A., not available; $(\boldsymbol{\Delta})$, significant increase with respect to the control group $(\boldsymbol{\nabla})$, significant reduction with respect to the control group; $(\bullet)$, without significant differences with respect to the control group; MDA, malondialdehyde.

jejunum (10\% and 7\%, respectively) in birds fed with $1.8 \mathrm{~g} / \mathrm{kg}$ of extract compared to the control group. The length of the ileum and caecum also increased $(8.3 \%$ and $11.9 \%$, respectively) in birds fed with the highest concentration of grape seed extract. Finally, the digestibility of phenolic compounds increased at day 42 between $60 \%$ to $69 \%$.

In another study performed by Goñi et al [71], the effect of including 5, 15, and $30 \mathrm{~g}$ of grape pomace and $200 \mathrm{mg}$ of a-acetate of tocopherol/kg in a basal diet during $21 \mathrm{~d}$ on productive parameters, protein and amino acid digestibility, antioxidant activity of the diet, blood serum, excreta, and lipid oxidation of meat during storage under refrigeration was investigated. The results showed that the inclusion of grape pomace in the diet increased the antioxidant activity of the diets and excreta (33.4\% and $15.8 \%$, respectively), although it did not affect antioxidant activity in serum, weight gain $(1.84 \mathrm{~kg})$, feed intake $(0.86 \mathrm{~kg})$, nutritional efficiency (1.3), or the ileal digestibility of essential and non-essential amino acids. Regarding the consumption and digestibility of phenolic compounds, an increase was observed (37.5\% and $56.4 \%$, respectively) in birds supplemented with $30 \mathrm{~g}$ of grape pomace $/ \mathrm{kg}$ compared to the control. In addition, in birds supplemented with grape pomace, the lipid oxidation values of breast and leg meat reduced (42.6\% and $30.2 \%$, respectively) at day 7 of refrigerated storage compared to the control. In a research developed by Sohaib et al [72], the influence of dietary quercetin $(100,200$, and $300 \mathrm{mg} / \mathrm{kg})$ in combination with $\alpha$-tocopherol $(150,225$, and $300 \mathrm{mg} / \mathrm{kg}$ ) during $6 \mathrm{wk}$, on lipid stability of breast meat (male broiler) was evaluated. The results showed that supplementation with $300 \mathrm{mg}$ quercetin $+300 \mathrm{mg}$ a-tocopherol/kg exhibited lowest lipid oxidation values ( $<0.3 \mathrm{mg}$ malondialdehyde $/ \mathrm{kg})$ in meat, as well as high antioxidant activity (antiradical DPPH', $>50 \%$ of inhibition; and ferric reducing antioxidant power, $>500$ $\mu \mathrm{mol} \mathrm{Fe}{ }^{+2}$ ), which could be associated to the highest values of total phenolic (>100 $\mathrm{mg}$ gallic acid equivalent [GAE]/g), quercetin $(16.4 \mathrm{mg} / \mathrm{kg})$ and $\alpha$-tocopherol $(38 \mathrm{mg} / \mathrm{kg})$ content.

In another research study conducted by Rupasinghe et al [73], the absorption and distribution of quercetin metabolites in plasma, various tissues, and excreta were evaluated in broilers supplemented with quercetin powder $(0,50,150$, and 300 $\mathrm{mg} / \mathrm{kg}$ of body weight/d), apple skin extract (50 and $150 \mathrm{mg}$ of total phenol content/kg of body weight/d), and dried apple skin meal (50 mg of total phenol content/kg of body weight/d) for $3 \mathrm{~d}$. Before supplementation, liquid chromatographytandem mass spectrometry (LC-MS/MS) was performed to identify the phenolic compounds present in the apple powder and apple extract. Quercetin glycosides such as quercetin- 
3-O-galactoside, quercetin-3-O-glucoside, quercetin-3-Orhamnoside, quercetin-3-O-rutinoside, and quercetin aglycone were found. The results showed that chickens fed with quercetin powder and apple skin extract at the tested concentrations had quercetin (in glycosylated form more than in aglycone form) in their excreta and plasma as well as in their duodenum and liver; this flavonoid was not detected in the control group. In addition, quercetin was found in chicken meat (breast and leg) supplemented with quercetin powder (300 $\mathrm{mg} / \mathrm{kg}$ ) and apple skin extract (150 and $200 \mathrm{mg} / \mathrm{kg}$ ) but was not found in the control group. This study shows that quercetin and its glycosides can be absorbed in broilers and, similar to what occurs in humans, are subjected to glucuronidation, sulfation, and methylation once absorbed.

In summary, these results show that phenolic compounds in their glycosylated form, once released from the food matrix, can be absorbed in the intestine by sugar transporters to the enterocyte (through the $\beta$-D-glucoside group) and metabolized by broiler chickens, subsequently exerting a pharmacological effect on birds. Meanwhile, phenolic compounds in free form (aglycone) present a low absorption because they lack sugar $[74,75]$.
Additionally, Cherian et al [76] evaluated the effect of Artemisia annua dried leaves ( $2 \%$ and $4 \%$ ) on digesta $\mathrm{pH}$ and muscle lipid oxidation, and phenolic distribution in dark and white meat of broiler (from day 14 through 42). The results showed that supplementation with Artemisia annua reduced $\mathrm{pH}$ values of ceca and ileal digesta, and lipid oxidation (thiobarbituric acid reactive substances) in the thigh muscle, which was associated to the increase of phenolic compounds (15.8\%) when compared with the control. However, the presence of phenolic compounds in the breast was not affected. In another study, Okarini et al [77] reported the presence of phenolic compounds (68.6, 65.6, and 64.4, respectively) in breast meat of Bali indigenous chicken (20 wk-old), spent laying hen (76 wk-old) and broiler (5 wk-old). Moreover, Vargas-Sánchez et al [78] studied the effect of Pleurotus ostreatus powder (1\% and $2 \%$ ) in Japanese quail diet (35 d) to increase the total antioxidant activity of their meat. At day 35 , the birds were slaughtering and whole breast removal, and then stored $\left(4^{\circ} \mathrm{C}\right.$ during $15 \mathrm{~d}$ ). Each sampling day, an aqueous extract was obtained from the breast an analyzed. The results showed that quails fed with Pleurotus ostreatus powder had the highest total phenolic and flavonoid content (>20 mg GAE/g, and

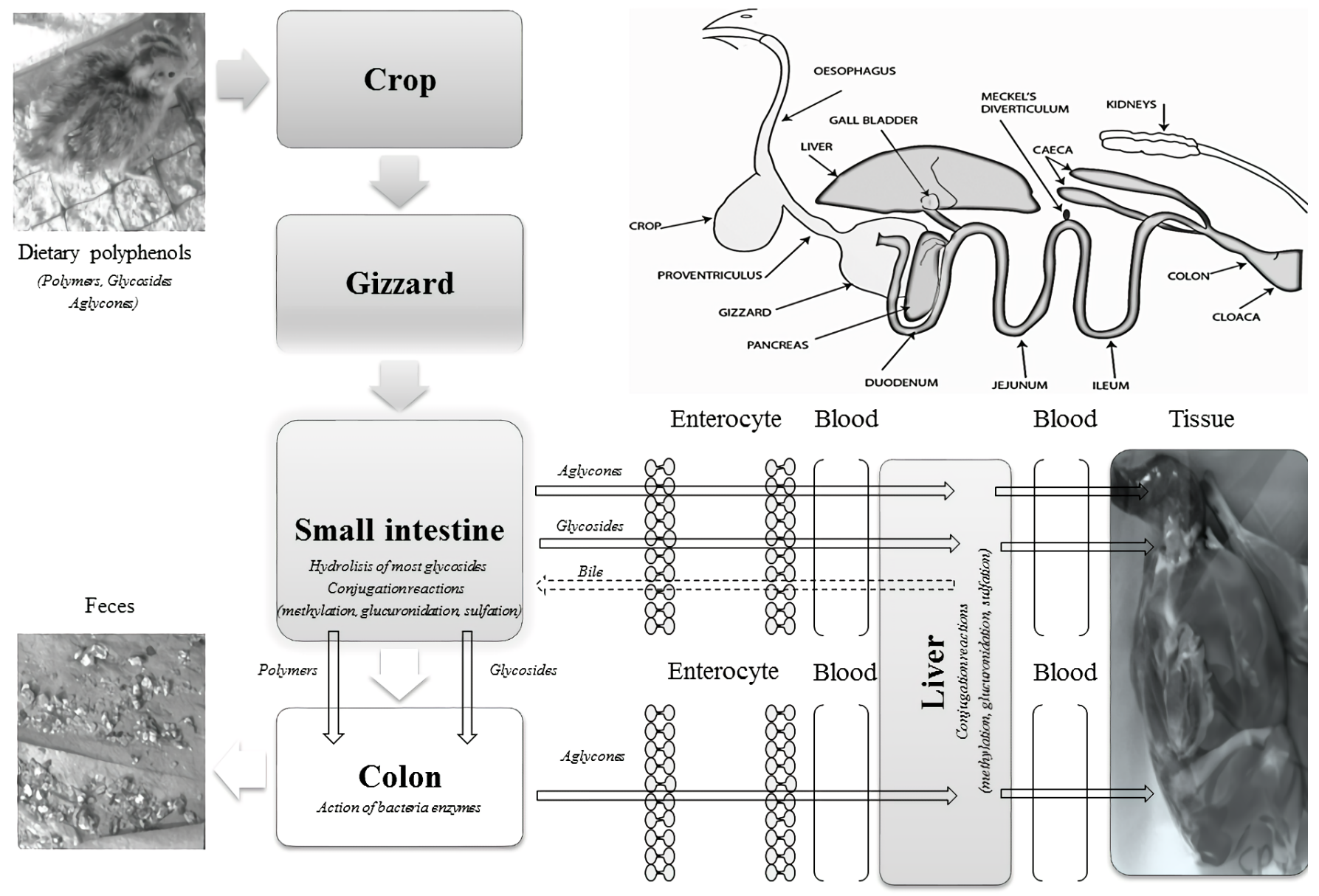

Figure 1. Schematic of dietary polyphenol transport to quail muscle (Addapted from: Ao et al [69]; Brenes et al [79]; Poultry-Hub [80]). 
$>15 \mathrm{mg}$ quercetin equivalents/g, respectively), as well as antiradical activity $\left(\mathrm{DPPH}^{\circ}\right.$ and $\left.\mathrm{ABTS}^{\circ}\right)$ when compared with control. The Figure 1 summarizes one of the metabolic absorption mechanisms of polyphenols in the quail diet.

\section{CONCLUSION}

The inclusion of natural ingredients in the diet of Japanese quail such as medicinal herbs, plants, vegetables, spices, seeds, worms, bee products, certain chemical compounds, and edible fungi has the potential to improve carcass and meat quality through reducing oxidative stress. However, this effect depends on the concentration of ingredients and on the type and/or conformation of the compounds present. In addition, these factors can improve or limit the absorption and metabolism of active compounds, enabling or disabling them from acting an antioxidant or antimicrobial agents. Furthermore, high concentrations of certain natural ingredients in the diet can possibly have adverse effects on quail carcasses and meat.

\section{CONFLICT OF INTEREST}

We certify that there is no conflict of interest with any financial organization regarding the material discussed in the manuscript.

\section{ACKNOWLEDGMENTS}

The authors like to thank ATISA for financial support, and Vargas-Sanchez gratefully acknowledges the fellowship received from CONACyT $(2015,1 ; 290941)$ for his postdoctoral work. Ibarra-Arias FJ is an employee of Alta Tecnología Industrial para la Salud Animal, S.A. de C.V.

\section{REFERENCES}

1. Poultry meat [Internet]. Mexico City, Mexico: Agro-food and fisheries information service; 2016 [cited 2018 Dec 11]. Available from: https://www.gob.mx/cms/uploads/attachment/ file/200631/Panorama_Agroalimentario_Avicultura_Carne_ 2016.pdf

2. Livestock and Poultry: World Markets and Trade [Internet]. Ithaca, NY, USA: United States Department of Agriculture; 2018 [cited 2018 Dec 11] Available from: https://apps.fas.usda. gov/psdonline/circulars/livestock_poultry.pdf

3. Gateway to Poultry Production and Products [Internet]. Rome, Italy: Food and Agriculture Organization; 2018 [cited 2018 Dec 11]. Available from: http://www.fao.org/poultry-productionproducts/production/poultry-species/en/

4. Cardozo-Jiménez D, Rebollar-Rebollar S, Rojo-Rubio R. Productivity and profitability of quail (Coturnix coturnix japonica) production in the south of Mexico State . Rev Mex Agroneg 2008;22:517-25.

5. Vali N. The Japanese quail: a review. Int J Poult Sci 2008;7: 925-31.

6. Alkan S, Karabag K, Galic A, Karsli T, Balcioglu MS. Determination of body weight and some carcass traits in Japanese quails (Coturnix coturnix japonica) of different-lines. Kafkas Univ Vet Fak Derg 2010;16:277-80.

7. Russian Federation Meat Sector Review [Internet]. Rome, Italy: Food and Agriculture Organization of the United Nations; 2014 [cited 2018 Dec 11]. Available from: http://www.fao. org/3/a-i3533e.pdf

8. Portillo-Loera JJ, Ríos-Rincón FG, Castro-Tamayo CB, AnguloMontoya C, Contreras-Pérez G. Carcass characteristics in mixed groups of Japanese quail (Coturnix coturnix japonica) in fattening slaughtered at different ages. Rev Cient FCV-Luz 2014;24:164-71.

9. Minvielle, F. The future of Japanese quail for research and production. World's Poult Sci J 2004;60:500-7. https://doi. org/10.1079/WPS200433

10. Díaz-Cuellar D, Juárez E, Maffei Valero MA, Morón-Fuenmayor O, González L, Morales J. Feeding of fattening quails (Coturnix coturnix japónica) based earthworm flour in two proteic levels. Agric Andina 2009;17:3-18.

11. National Research Council. Nutriment requirements of poultry. Nutrient requirements of ring-necked Pheasants, Japanese quail, and Bobwhite quail. 9th Rev. Whashington, DC, USA: National Academy Press; 1994.

12. Altine S, Sabo MN, Muhammad N, Abubakar A, Saulawa LA. Basic nutrient requirements of the domestic quails under tropical conditions: a review. World Sci News 2016;49:223-35.

13. Morón-Fuenmayor OE, Díaz D, Pietrosemoli S, et al. Effect of earthworm (Eiseni spp) meal inclusion on dressing and physical-chemical characteristics of quail meat (Coturnix coturnix japonica). Rev Fac Agro 2008;25:674-84.

14. Obregón JF, Bell C, Elenes I, Estrada A, Portillo JJ, Ríos FG. Effect of the cooking of chickpea (Cicer arietinum L.) discard in the productive response and carcass yield of the Japanese fattening quail (Coturnix coturnix japonica). Rev Cub Cien Agric 2012;46:169.

15. Smet K, Raes K, Huyghebaert G, Haak L, Arnouts S, De Smet S. Lipid and protein oxidation of broiler meat as influenced by dietary natural antioxidant supplementation. Poult Sci 2008;87:1682-8. https://doi.org/10.3382/ps.2007-00384

16. Genchev A, Mihaylova G, Ribarski S, Pavlov A, Kabakchiev M. Meat quality and composition in Japanese quails. Trakia J Sci 2008;6:72-82.

17. Hamm D, Ang CYW. Nutrient composition of quail meat from three sources. J Food Sci 1982;47:1613-14. https://doi.org/10. 1111/j.1365-2621.1982.tb04994.x

18.Du M, Ahn DU. Effect of dietary conjugated linoleic acid on the growth rate of live birds and on the abdominal fat content 
and quality of broiler meat. Poult Sci 2002;81:428-33. https:// doi.org/10.1093/ps/81.3.428

19.Lee TT, Ciou JY, Chiang CJ, Chao YP, Yu B. Effect of Pleurotus eryngii stalk residue on the oxidative status and meat quality of broiler chickens. J Agric Food Chem 2012;60:11157-63. https://doi.org/10.1021/jf302740h

20.Williams, P. Nutritional composition of red meat. Nutr Diet 2007;64:S113-S19. https://doi.org/10.1111/j.1747-0080.2007. 00197.xz

21.Cullere M, Tasoniero G, Giaccone V, Acuti G, Marangon A, Dalle Zotte A. Black soldier fly as dietary protein source for broiler quails: Meat proximate composition, fatty acid and amino acid profile, oxidative status and sensory traits. Animal 2018;12:640-7. https://doi.org/10.1017/S1751731117001860

22. Ravindran V. Nutrition of meat animals. Poultry. In: Dikeman M, Devine C, editors. Encyclopedia of meat sciences (Second Edition). USA. Oxford, UK: Academic Press; 2014. p. 436-70.

23. Hoke IM, Buege DR, Ellefson W, Maly E. Nutrient and related food composition of exported Australian lamb cuts. J Food Compost Anal 1999;12:97-109.

24. Torrescano-Urrutia GR, Sánchez-Escalante A, Vásquez-Palma MG, Varguez-Pech AF, Vargas-Sánchez RD, Pardo-Guzmán DA. Estimation of the degree of marbling of sonoran beef carcasses using different methods: image analysis, USDA evaluation, and solvent extraction. Biotecnia 2018;19:34-9.

25. Botsoglou N, Papageorgiou G, Nikolakakis I, et al. Effect of dietary dried tomato pulp on oxidative stability of Japanese quail meat. J Agric Food Chem 2004;52:2982-8. https:/doi. org/10.1021/jf030748b

26. Khatibjoo A, Kermanshahi H, Golian A, Zaghari M. The effect of n-6/n-3 fatty acid ratios on broiler breeder performance, hatchability, fatty acid profile and reproduction. J Anim Physiol Anim Nutr 2018;102:986-98. https://doi.org/10.1111/jpn.12904

27. Mehdipour Z, Afsharmanesh M, Sami M. Effects of dietary synbiotic and cinnamon (Cinnamomum verum) supplementation on growth performance and meat quality in Japanese quail. Livest Sci 2013;154:152-7. https://doi.org/10.1016/j.livsci. 2013.03.014

28. Wattanachant S, Benjakul S, Ledward, DA. Composition, color, and texture of Thai indigenous and broiler chicken muscles. Poult Sci 2004;83:123-8. https://doi.org/10.1093/ps/83.1.123

29. Santoso U, Fenita Y, Kususiyah K, Widiantoro O, Kadarsih S. The effect of medicinal herb on fat deposition, meat composition, amino acid and fatty acid composition of broiler meats. J Indones Trop Anim Agric 2018;43:54-65. https://doi.org/10. 14710/jitaa.43.1.54-65

30. Estévez M. Oxidative damage to poultry: from farm to fork. Poult Sci 2015;94: 1368-78. https://doi.org/10.3382/ps/pev094

31. Van Horne PLM, Achterbosch TJ. Animal welfare in poultry production systems: impact of EU standards on world trade. World's Poult Sci J 2008;64:40-52. https://doi.org/10.1017/ S0043933907001705
32. Remignon $\mathrm{H}$, Mills AD, Guemene D, et al. Meat quality traits and muscle characteristics in high or low fear lines of Japanese quails (Coturnix japonica) subjected to acute stress. Br Poult Sci 1998;39:372-8. https://doi.org/10.1080/00071669888926

33. Shrivastav AK, Panda B. A review of quail nutrition research in India. World's Poult Sci J 1999;55:73-81. https://doi.org/10. 1079/WPS19990006

34. González VA, Rojas GE, Aguilera AE, Flores-Peinado SC, et al. Effect of heat stress during transportation and rest before slaughter, on the metabolic profile, blood gases and meat quality of quail. Int J Poult Sci 2007;6:397-402. http://dx.doi. org/10.3923/ijps.2007.397.402

35. Ali AS, Harrison AP, Jensen JF. Effect of some ante-mortem stressors on peri-mortem and post-mortem biochemical changes and tenderness in broiler breast muscle: a review. World's Poult Sci J 1999;55:403-14. https://doi.org/10.1079/ WPS19990028

36. Castellini C, Mugnai C, Dal Bosco A. Effect of organic production system on broiler carcass and meat quality. Meat Sci 2002; 60:219-25. https://doi.org/10.1016/S0309-1740(01)00124-3

37.Vargas-Sánchez RD, Torrescano-Urrutia GR, Ibarra-Arias FJ, Portillo-Loera JJ, Ríos-Rincón FG, Sánchez-Escalante A. Effect of dietary supplementation with Pleurotus ostreatus on growth performance and meat quality of Japanese quail. Livest Sci 2018;207:117-25. https://doi.org/10.1016/j.livsci. 2017.11.015

38. Butaye P, Devriese LA, Haesebrouck F. Antimicrobial growth promoters used in animal feed: effects of less well known antibiotics on gram-positive bacteria. Clin Microbiol Rev 2003;16:175-88. https://doi.org/10.1128/CMR.16.2.175-188. 2003

39. National Strategy of Action Against Resistance to Antimicrobials [Internet]. Mexico City, Mexico: Official Journal of the Federation, 2018 [cited 2018 Jun 1]. Available from: http:// dof.gob.mx/nota_detalle.php?codigo $=5525043 \&$ fecha $=05 /$ 06/2018

40. Yopasá-Arenas A, Fostier AH. Exposure of Brazilian soil and groundwater to pollution by coccidiostats and antimicrobial agents used as growth promoters. Sci Total Environ 2018;644: 112-21. https://doi.org/10.1016/j.scitotenv.2018.06.338

41.Guo FC, Williams BA, Kwakkel RP, et al. Effects of mushroom and herb polysaccharides, as alternatives for an antibiotic, on the cecal microbial ecosystem in broiler chickens. Poult Sci 2004;83:175-82. https://doi.org/10.1093/ps/83.2.175

42. Falowo AB, Fayemi PO, Muchenje, V. Natural antioxidants against lipid-protein oxidative deterioration in meat and meat products: a review. Food Res Int 2014;64:171-81. https://doi. org/10.1016/j.foodres.2014.06.022

43.Löliger J, Wille HJ. Natural antioxidants. Oils \& Fats Int 1993;9: 18-22.

44. Karre L, Lopez K, Getty KJ. Natural antioxidants in meat and poultry products. Meat Sci 2013;94:220-27. https://doi.org/ 
10.1016/j.meatsci.2013.01.007

45. Codex Alimentarius Commission. Procedural manual. TwentySixth edition. Rome, Italy: Food and Agricultural Organization of the United Nations; 1995. p. 23.

46. Good Practices for the Feed Insdustry [internet]. Rome, Italy: Food Agriculture Oranization of the United Nations; 2014 [cited 2018 Sept 1]. Available from: http://www.fao.org/docrep/ 012/i1379e/i1379e.pdf

47.Denli M, Okan F, Uluocak AN. Effect of dietary supplementation of herb essential oils on the growth performance, carcass and intestinal characteristics of quail (Coturnix coturnix japonica). S Afr J Anim Sci 2004;34:174-9.

48.Sengül T, Yurtseven S, Cetin M, Kocyigit A, Sögüt B. Effect of thyme (T. vulgaris) extracts on fattening performance, some blood parameters, oxidative stress and DNA damage in Japanese quails. J Anim Feed Sci 2008;17:608-20. https:// doi.org/10.22358/jafs/66689/2008

49. Chantiratikul A, Chantiratikul P, Sangdee A, Maneechote U, Bunchasak C, Chinrasri O. Performance and carcass characteristics of Japanese quails fed diets containing Wolffia Meal [Wolffia globosa (L). Wimm.] as a protein replacement for soybean meal. Int J Poult Sci 2010;9:562-6. http://dx.doi.org/ 10.3923/ijps.2010.562.566

50. Khaksar V, Van Krimpen M, Hashemipou, H, Pilevar M. Effects of thyme essential oil on performance, some blood parameters and ileal microflora of Japanese quail. J Poult Sci 2012;49:106-110. https://doi.org/10.2141/jpsa.011089

51.Ghazaghi M, Mehri M, Bagherzadeh-Kasmani F. Effects of dietary Mentha spicata on performance, blood metabolites, meat quality and microbial ecosystem of small intestine in growing Japanese quail. Anim Feed Sci Technol 2014;194:8998. https://doi.org/10.1016/j.anifeedsci.2014.04.014

52.Mehri M, Sabaghi V, Bagherzadeh-Kasmani F. Mentha piperita (peppermint) in growing Japanese quails diet: Performance, carcass attributes, morphology and microbial populations of intestine. Anim Feed Sci Technol 2015;207:104-11. https:// doi.org/10.1016/j.anifeedsci.2015.05.021

53. Kara K, Şentürk M, Guclu BK, Sariözkan S, Eren M. Effect of catechins on fattening performance, meat quality, some antioxidant and blood parameters and fattening costs in Japanese quail (Coturnix coturnix japonica). Br Poult Sci 2016;57:52230. https://doi.org/10.1080/00071668.2016.1174977

54.Mnisi CM, Matshogo TB, van Niekerk R, Mlambo V. Growth performance, haemo-biochemical parameters and meat quality characteristics of male Japanese quails fed a Lippia javanicabased diet. S Afr J Anim Sci 2017;47:661-71. http://dx.doi. org/10.4314/sajas.v47i5.9

55.Mnisi CM, Mlambo V. Growth performance, haematology, serum biochemistry and meat quality characteristics of Japanese quail (Coturnix coturnix japonica) fed canola meal-based diets. Anim Nutr 2018;4:37-43. https://doi.org/10.1016/ j.aninu.2017.08.011
56. Güler T, Ertas ON, Ciftci M, Dalkilic B. The effect of coriander seed (Coriandrum sativum L.) as diet ingredient on the performance of Japanese quail. S Afr J Anim Sci 2005;35:261-7. http:// dx.doi.org/10.4314/sajas.v35i4.3969

57.Denli M, Cankaya S, Silici S, Okan F, Uluocak AN. Effect of dietary addition of Turkish propolis on the growth performance, carcass characteristics and serum variables of quail (Coturnix coturnix japonica). Asian-Australas J Anim Sci 2005; 18:848-54. https://doi.org/10.5713/ajas.2005.848

58. Canogullari S, Baylen M, Sahinler N, Sahin A. Effects of propolis and pollen supplementations on growth performance and body components of Japanese quails (Coturnix coturnix japonica). Arch Geflugel 2009;73:173-8.

59. Sahin K, Onderci M, Sahin N, Gursu MF, Kucuk O. Dietary vitamin $\mathrm{C}$ and folic acid supplementation ameliorates the detrimental effects of heat stress in Japanese quail. J Nutr 2003;133:1882-6. https://doi.org/10.1093/jn/133.6.1882

60.Sahin K, Onderci M, Sahin N, Gursu MF, Khachik F, Kucuk O. Effects of lycopene supplementation on antioxidant status, oxidative stress, performance and carcass characteristics in heat-stressed Japanese quail. J Therm Biol 2006;31:307-12. https://doi.org/10.1016/j.jtherbio.2005.12.006

61.Asadi-Dizaji A, Shahryar HA, Shaddel-Tili A, Maheri-Sis N, Ghiasi-Ghalehkandi J. Effect of Common mushroom (Agaricus bisporus) Levels on Growth Performance and Carcass yields of Japanese quails (Coturnix coturnix Japonica). Bull Env Pharmacol Life Sci 2014;3:1-5.

62. Mehri M, Sabaghi V, Bagherzadeh-Kasmani F. Mentha piperita (peppermint) in growing Japanese quails' diet: Serum biochemistry, meat quality, humoral immunity. Anim Feed Sci Technol 2015;206:57-66. https://doi.org/10.1016/j.anifeedsci. 2015.05.022

63. Adriani L, Roni P, Hendronoto A, Lengkey W, Bagus P. Using bay leaf mill (Syzygium polyanthum, Wight) in ration on fat and cholesterol levels of quail meat (Coturnix coturnix japonica). In: SAADC 2011 strategies and challenges for sustainable animal agriculture-crop systems, Volume III: full papers. Proceedings of the 3rd International Conference on sustainable animal agriculture for developing countries; 26-29 July: Nakhon Ratchasima, Thailand; 2011.pp. 518-21.

64. Yalcin H, Konca Y, Durmuscelebi F. Effect of dietary supplementation of hemp seed (Cannabis sativa L.) on meat quality and egg fatty acid composition of Japanese quail (Coturnix coturnix japonica). J Anim Physiol Anim Nutr 2018;102:131-41. https:// doi.org/10.1111/jpn.12670

65. Viveros A, Chamorro S, Pizarro M, Arija I, Centeno C, Brenes A. Effects of dietary polyphenol-rich grape products on intestinal microflora and gut morphology in broiler chicks. Poult Sci 2011;90:566-78. https://doi.org/10.3382/ps.2010-00889

66. Surai PF. Polyphenol compounds in the chicken/animal diet: from the past to the future. J Anim Physiol Anim Nutr 2014; 98:19-31. https://doi.org/10.1111/jpn.12070 
67.Zhou YB, Wan XC, Shang YY, et al. Polyphenol content of plasma and litter after the oral administration of green tea and tea polyphenols in chickens. J Agric Food Chem 2012; 60:1619-27. https://doi.org/10.1021/jf2039789

68. Jimenez-Ramsey LM, Rogler JC, Housley TL, Butler LG, Elkin RG. Absorption and distribution of 14C-labeled condensed tannins and related sorghum phenolics in chickens. J Agric Food Chem 1994;42:963-7. https://doi.org/10.1021/jf00040a024

69. Ao T, Cantor AH, Pescatore AJ, Pierce JL, Dawson KA. Effects of citric acid, alpha-galactosidase and protease inclusion on in vitro nutrient release from soybean meal and trypsin inhibitor content in raw whole soybeans. Anim Feed Sci Technol 2010;162:58-65. https://doi.org/10.1016/j.anifeedsci.2010. 08.014

70. Brenes A, Viveros A, Goñi I, Centeno C, Saura-Calixto F, Arija I. Effect of grape seed extract on growth performance, protein and polyphenol digestibilities, and antioxidant activity in chickens. Spanish J Agric Res 2010;8:326-33.

71. Goñí I, Brenes A, Centeno C, Viveros A, et al. Effect of dietary grape pomace and vitamin $\mathrm{E}$ on growth performance, nutrient digestibility, and susceptibility to meat lipid oxidation in chickens. Poult Sci 2007;86:508-16. https://doi.org/10.1093/ps/86.3.508

72.Sohaib M, Butt MS, Shabbir MA, Shahid, M. Lipid stability, antioxidant potential and fatty acid composition of broilers breast meat as influenced by quercetin in combination with a-tocopherol enriched diets. Lipids Health Dis 2015;14:61. https://doi.org/10.1186/s12944-015-0058-6

73. Rupasinghe HP, Ronalds CM, Rathgeber B, Robinson RA. Absorption and tissue distribution of dietary quercetin and quercetin glycosides of apple skin in broiler chickens. J Sci
Food Agric 2010;90:1172-8. https://doi.org/10.1002/jsfa.3944

74. Hollman PC, De Vries JH, van Leeuwen SD, Mengelers M), Katan MB. Absorption of dietary quercetin glycosides and quercetin in healthy ileostomy volunteers. Am J Clin Nutr 1995;62:1276-82. https://doi.org/10.1093/ajcn/62.6.1276

75. Yatao X, Saeed M, Kamboh A, et al. The potentially beneficial effects of supplementation with hesperidin in poultry diets. World's Poult Sci J 2018;74:265-76. https://doi.org/10.1017/ S0043933917001088

76. Cherian G, Orr A, Burke IC, Pan W. Feeding Artemisia annua alters digesta $\mathrm{pH}$ and muscle lipid oxidation products in broiler chickens. Poult Sci 2013;92:1085-90. https://doi.org/10.3382/ ps.2012-02752

77. Okarini IA, Purnomo H, Radiati LE. Proximate, total phenolic, antioxidant activity and amino acids profile of Bali indigenous chicken, spent laying hen and broiler breast fillet. Int J Poult Sci 2013;12:415-20. http://dx.doi.org/10.3923/ijps.2013.415. 420

78. Vargas-Sánchez RD, Velásquez-Jiménez D, Torrescano-Urrutia GR, et al. Total antioxidant activity in japanese quail (Coturnix coturnix japonica) breast, fed a supplemented diet of edible mushrooms. Biotecnia 2018; 20:43-50.

79. Brenes A, Viveros A, Chamorro S, Arija I. Use of polyphenolrich grape by-products in monogastric nutrition. A review. Anim Feed Sci Technol 2016;211:1-17. https://doi.org/10.1016/ j.anifeedsci.2015.09.016

80.Poultry-Hub. Digestive system [Internet]. Poultry CRS; 2008 [Cited 2018 Dec 11]. Available from: http://www.poultryhub. org/physiology/body-systems/digestive-system/ 